\title{
Mapping the Molecular Tree of Life using Assembly Spaces
}

Yu Liu, Cole Mathis, Stuart Marshall, Leroy Cronin*

School of Chemistry, the University of Glasgow, University Avenue, Glasgow G12 8QQ, UK.

Email:lee.cronin@glasgow.ac.uk

The mapping of chemical space by the enumeration of graphs generates an infinite number of molecules, yet the experimental exploration of known chemical space shows that it appears to become sparser as the molecular weight of the compounds increases. What is needed is a way to explore chemical space that exploits the information encoded in known molecules to give access to unknown chemical space by building on the common conserved structures found in related families of molecules. Molecular assembly theory provides an approach to explore and compare the intrinsic complexity of molecules by the minimum number of steps needed to build up the target graphs, and here we show this can be applied to networks of molecules to explore the assembly properties of common motifs, rather than just focusing on molecules in isolation. This means molecular assembly theory can be used to define a tree of assembly spaces, allowing us to explore the accessible molecules connected to the tree, rather than the entire space of possible molecules. This approach provides a way to map the relationship between the molecules and their common fragments and thus measures the distribution of structural information collectively embedded in the molecules. We apply this approach to prebiotic chemistry, specifically the construction of RNA, and a family of opiates and plasticizers, as well as to gene sequences. This analysis allows us to quantify the amount of external information needed to assemble the tree and identify and predict new components in this family of molecules, based on the contingent information in the assembly spaces. 


\section{Introduction}

Chemical space is populated by molecules, oligomers, and polymers which can be characterized by their molecular composition, precise formula, graph representation, structural identity and reactivity $(1,2)$. A major feature of the unconstrained search of chemical space lies with the fact that chemical space can be occupied by discrete molecules, but it is infinitely large according to the laws of quantum mechanics. In fact, chemical space, if it is explored by the enumeration of graphs, can be enumerated to give an unimaginably vast number of at least $10^{60}$ small organic molecules $(1,3,4)$. It could be argued this is not realistic as many of these molecules might be unstable or inaccessible synthetically, so this means the unbound search of such a large space is likely to be problematic. However, when exploring biochemistry, only a few hundred different types of 'unique' small molecules are needed by the simplest living organisms $(1,5)$, indicating that the chemical space relevant to biology (or Earth biology) is merely a tiny fraction of chemical space that is possible $(6,7)$. The issue therefore arises about how the space of molecules can be effectively searched $(1,2$, 8) and what constrains molecules to being possible (2,9-11), because for most use cases the interesting areas of chemical space will be vanishingly small compared to the space of possibilities $(9,12-15)$.

One way to both imagine and explore the universe of molecules is to define and construct a chemical space as a fixed dataset, e.g., GDB-17 database (16, 17), DrugBank (18) and PubChem (19), and then navigate the dataset using various descriptors, e.g., the 42 molecular quantum numbers (20), molecular fingerprints $(21,22)$, and values calculated from the principal component analysis for a combination of various descriptors (23). Although searching these fixed chemical spaces is straightforward as molecules with similar chemical and biological properties will be close to each other, it is computationally expensive and 
inefficient, because it requires exhaustively enumerating (or with optimization algorithms such as Bayesian optimization (24)) and screening molecules for desirable properties. Similarly, it is possible to iteratively generate chemical subspaces, followed by filtering unwanted structures, until desired molecules are obtained. This can be done by using a genetic algorithm $(25,26)$, using extrapolation techniques such as ACSESS (Algorithm for Chemical Space Exploration with Stochastic Search) $(27,28)$, or even employing human intuition (29). However, given the relative sizes of possible chemical space compared to the number of interesting molecular structures, these approaches are like different methods for searching for a needle in a haystack, and therefore it is not clear how comprehensive any of these approaches can be in principle.

Recently, machine learning and statistical techniques have been introduced into the navigation of chemical space $(30,31)$. For example, hundreds of thousands of existing chemical structures were used to train a deep neural network so that each molecule (either existing or generated) can be assigned to a set of discrete coordinates in the continuous latent space of the neural network. Navigating within this discrete chemical space corresponds to navigating in the continuous latent space, which is much easier computationally (32). Alternatively, a convolutional neural network can be used to train directly on graph representations of molecules to infer their molecular features, generating the so-called neural graph fingerprints and these can have a better predictive performance over the existing handcrafted fingerprints in some applications (33). These approaches offer a significant improvement over raw enumeration and filtering because they compress the search space, offering the opportunity to efficiently navigate the large space of possible compounds to find useful subspaces. Unfortunately, while neural networks might make the navigation of chemical spaces more efficient, the chemical space is treated as a black box. The intrinsic 
properties of chemical space (e.g., hierarchical information among molecular structures) are therefore obscured. This is a significant problem since the structure of chemical space is thought to encode a great deal of important contingent information $(1,2)$. This is because the current structure of observable chemical space is highly connected to the chemical space of biology, which itself has been selected and explored using evolution over the past 4 billion years on Earth $(1,10,11,34)$. Our hypothesis is that the information used to assemble the molecules found in biology was produced by random selection and is conserved and reused by the various biochemical pathways across all biology. This means that a lot of chemical space can be encoded by what is accessible from these trees and we are interested in exploring how this might be mapped, constructed, and searched algorithmically but also updated experimentally.

In this work we present a new way of constructing a space of related molecules that not only allows us to understand how accessible chemical space is nucleated from the molecules produced and selected by evolution, but also can allow us to predict which spaces of molecules are both accessible and important, functional, and potentially novel. This is because it is easy to confuse what chemical spaces might be imagined, allowed by the laws of physics and chemistry compared to what is accessible based on known motifs. Our approach defines chemical space based upon this accessibility and uses information that is encoded within the tree of related molecules to help define the most accessible avenues for exploration. Further, by exploring these trees, with information from other sources about what molecules are synthetically feasible, it will be possible to develop new routes to structure expansion that encode the transformations of chemical synthesis. 


\section{Establishing the theory of Assembly Spaces of Molecules}

Assembly theory was developed to quantify the amount of information required to construct or select a given molecule in any non-trivial abundance, and hence can be used to determine if a given molecule was produced using external information, and for exploring biosignatures $(35,36)$. Briefly, assembly theory quantifies the constraints required to produce a molecule by measuring the minimum number of steps to produce the molecular graph. First, we demonstrate that this can not only be done for single molecules, but also for groups and networks of molecules whereby commonalities in the building blocks can be exploited to find a common set that allows access to a given set of molecules. The common fragments that can be used to assemble the set of molecules define an assembly space. By exploiting this consequence of assembly theory, we demonstrate how it can be used to explore the structure of chemical space and how assembly spaces can provide compressed representations of chemical spaces that offer a new way to generate new compounds by exploiting (rather than obscuring) hierarchical relationships between compounds. Here we elaborate on the concept of assembly theory and develop the terminologies, notations, and theoretical calculations in the context of molecules, by taking the molecule adenine (represented by a graph where the nodes represent atoms while the edges represent bonds) as an example, see Fig. 1.

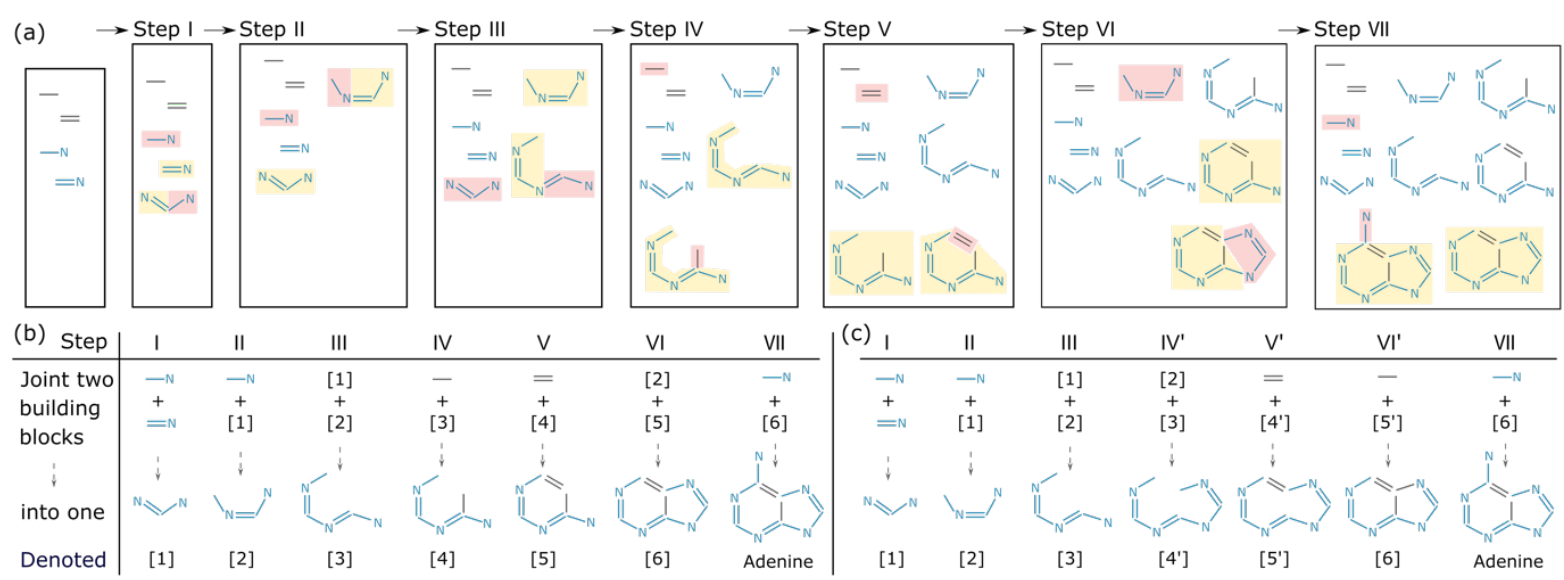

Fig. 1. (a) One assembly pathway of adenine. The surrounding box represents the assembly pool. The colored patches are just used to help the reader recognize which two building blocks are used to make the new one (note that the color schemes are independent for each assembly pool). (b) The sequential representation of the same assembly pathway as in (a). (c) Another assembly pathway of adenine, having the same number of steps as in (b). 
As mentioned above, Molecular Assembly Theory allows us to look at the minimum number of steps to construct a molecular graph by recursively using previously made structure. In general, we could choose any class of objects as the building blocks to construct the target, depending on the context. For example, we could choose nucleotides guanine (G), adenine (A), cytosine (C) and thymine (T) as the basic building blocks for DNA sequence related questions, or G, A, C and uracil (U) for RNA; we could choose monomers for polymer related questions; and we could choose either atoms or bonds as basic building blocks for molecules. For illustration we chose the four chemical bonds that make adenine, namely carbon-carbon single bond, carbon-carbon double bond, carbon-nitrogen single bond and carbon-nitrogen double bond, as the basic building blocks (note that we only consider nonhydrogen atoms, and any bond with a hydrogen atom attached is thus ignored). As a result, any of these chemical bonds and the molecular structures that will be produced in later steps are the assembly building block and we call the set of all assembly building blocks as the assembly pool (see Fig. 1a). In this assembly pool, any type of the assembly building block is assumed to have infinite instances.

One assembly step is precisely defined as the three sequential operations: (i) Take two assembly building blocks from the assembly pool; (ii) Join the two together in a way based on the particular system in question (in our context, that is, to superimpose certain atoms from the two building blocks to make a larger molecular structure); (iii) Put the new composite building block back into the assembly pool, and it will be considered as an assembly building block hereafter. Figure 1a shows 7 consecutive steps after which the target molecule adenine is constructed and appears in the assembly pool. One sequence of the assembly steps that can successfully construct the target molecule is defined as an assembly 
pathway of this molecule. Figure 1a shows the whole assembly pool for every step, and Fig.1b shows a simplified representation. In general, there are many different assembly pathways for one molecule (Fig. 1c shows another one for adenine). The number of assembly steps of an assembly pathway is defined as the assembly index of this pathway. Therefore, the index of either assembly pathway shown in Fig. 1a or $1 \mathrm{~b}$ is 7.

Assembly pathways of a molecule that have the minimum number of assembly steps, i.e., the shortest assembly pathways of a molecule, are of particular interest because they can provide a bound on the likelihood of spontaneous formation of the molecule in the absence of any informational system (note that there could be more than one shortest assembly pathways). The assembly index of the shortest assembly pathway(s) is defined to be the molecular assembly number (MA) of this molecule. The two assembly pathways shown in Fig. 1a and $1 \mathrm{~b}$ are the shortest (worked out by the algorithm we have developed, which will be introduced in the next section), and adenine's MA is thus 7. Lastly, we may use the term assembly space of a molecule in a more general sense to refer to all of the assembly building blocks and the associated relationships included in many or all assembly pathways of the molecule. If no ambiguity is raised, we may just say "pathway", "index" and "space" hereafter, for convenience.

\section{Representation of assembly pathway $\&$ index calculation}

In assembly pathways the order of certain steps can be switched without changing the size of the pathway. This leads to a combinatorial number of trivial pathways that all have the same number of steps. For instance, in Fig. 1, starting from building block [3], to make building block [6], we can either add a carbon-carbon single bond to [3], and then a carbon-carbon double bond, and lastly add building block [2] (just like the pathway shown in Fig. 1b); or we 
can add [2] first, and then a double bond, and lastly a single bond, which leads to another pathway (as shown in Fig. 1c). Conversely, order does matter when making some other building blocks. Again, taking Fig. 1b as an example, building block [1] must be made before [2] and [3] because [1] is used to make [2] and [3]; building block [2] must be made before [3] and [6] because [2] is used to make them. We can utilize these properties to represent assembly pathways without ambiguity, by focusing on the steps in which order matter. We call those special building blocks that define the hierarchical relationships among the chemical structures as the key assembly building blocks---equivalently, they are the assembly building blocks that are used more than once in the pathway---and the corresponding step as the key assembly step. Therefore, we can represent a pathway in terms of key steps, which eliminates all the trivial information. For example, for both the pathways shown in Fig. $1 \mathrm{~b}$ and 1c, the key building blocks are [1] and [2], so both can be represented identically, as shown in Fig. 2a.

In the key-step representation (Fig. 2a), although it is not immediately obvious how many key building blocks the final target molecule consists of, the numbers can be worked out based solely on this representation. As shown in Fig. 2b, we can explicitly write down the joint process for each key step, and then remove the building blocks that appear on both sides (as indicated by the double slashes). The building blocks left over constitute the target molecule non-repetitively, which are denoted as a multiset (37), as shown in Fig. 2c. That is to say, the target molecule adenine can be made from these chemical bonds and structures non-repetitively and by the least number of steps. In fact, the information of the basic building blocks (namely, individual bonds) is trivial (because it can be easily worked out by counting the number of bonds of the target molecule) and can thus be omitted. This simpler representation is shown in Fig. 2d. The key-step representation (Fig. 2a) and multiset 
representation (Fig. 2d) are one-to-one, i.e., either one can be worked out from the other without ambiguity. The key-step representation emphasizes the hierarchical relationship between building blocks, while the multiset representation emphasizes the information of constituents and provides compact summary of the assembly space associated with this pathway.
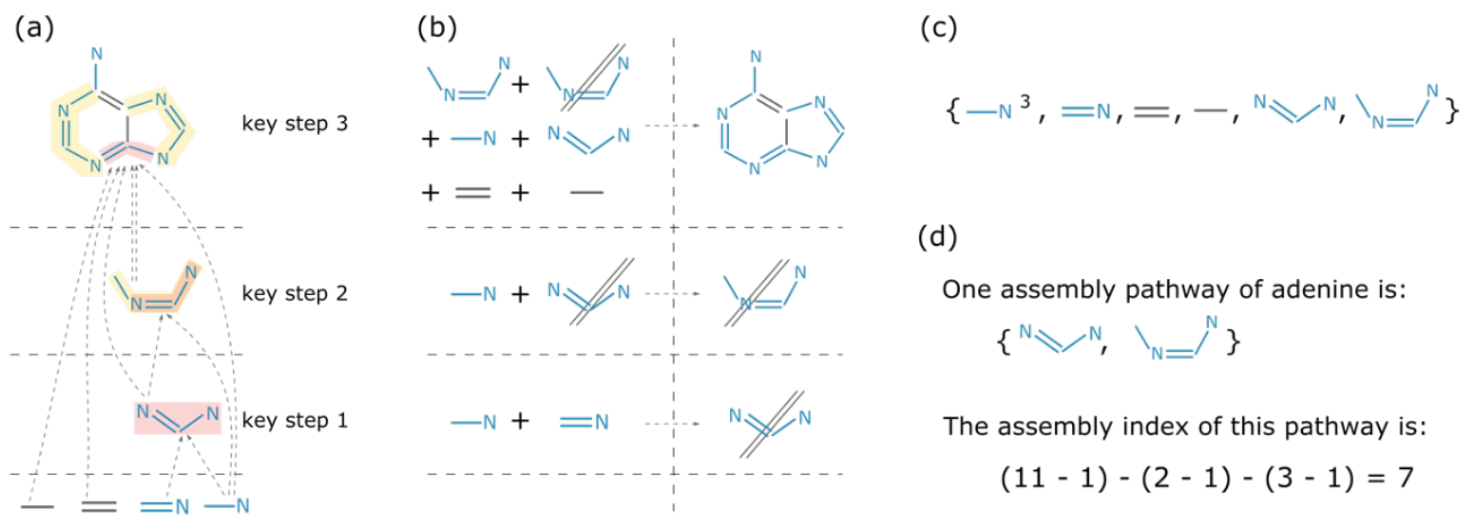

Fig. 2. (a) The key-step representation of the assembly pathway of adenine as the one shown in Fig. 1b. The pathway shown in Fig. 1c can also be represented by (a). The colored patches are just used to help the reader recognize the building blocks. (b) The joint process for each key step. (c) The multiset representation of the pathway. The superscript " 3 " is the multiplicity of this structure, that is, it appears 3 times on the left hand side of (b). If the multiplicity is 1, it is always omitted. (d) After filtering the trivial information about the individual bonds, we obtain the clean multiset representation of this pathway finally. Using this representation, the assembly index can be worked out easily, which is 7 (see the main text).

Lastly, based on the multiset representation, the index of this pathway can be easily worked out, as shown in Fig. 2d. The logic is: (i) The longest assembly pathway for a molecule is to join one bond for each step. As adenine has 11 bonds, we need 11-1=10 steps. (ii) By reusing one building blocks shown in the multiset representation, $x$ number of steps is saved, where $x$ is equal to the number of bonds of this building block minus 1. For the building block [2] (referring to Fig. 1b) for example, it saves 3-1=2 steps. Finally, we obtain the index of this pathway, equal to $10-1-2=7$, which is consistent with the result as we directly count the number of steps according to the definition (referring to Fig. 1b). An assembly pathway of a molecule does not necessarily correspond to a realistic sequence of chemical reactions that produce this molecule, or how biology / metabolism produces it, or how industry / technology synthesizes it. Instead, the shortest pathway shows how to make a molecule in the least steps 
in principle. No matter what methods or synthesis approaches are employed, there will be no other way shorter than this idealistic one. It thus reflects an intrinsic property of a molecule.

\section{Monte Carlo algorithm to compute the shortest assembly pathways}

In this section, we will introduce a Monte Carlo algorithm to compute the shortest assembly pathways of a molecule. A Monte Carlo algorithm was developed because the computational power required to solve the problem exactly is expected to be significant for large molecules. Notice that, this problem could be considered as a combination of two difficult classic problems: (i) the shortest addition chain problem (38-40) and (ii) the subgraph isomorphism problem which has been proven to be NP-complete $(41,42)$. Thus, it is at least as hard as NPcomplete (see SI section 2 for details). Since obtaining precise results is computationally expensive, we adopted the Monte Carlo approach (43) that allows us to efficiently calculate approximate results that are exact in the limit of infinite computational time (see SI section 3 for details).

First, we need to calculate a distribution that shows how many times a molecular structure or fragment is duplicated in the original molecule. Figure 3 a shows a part of the distribution for adenine, and three exemplified fragments. To obtain the full distribution, we would need to fragment the molecule in every possible way, however as the size of the molecule increases, the number of all possible fragmenting schemes grows rapidly, leading to a combinatorial explosion. So, in this step, we adopted the Monte Carlo approach where we (i) randomly fragment the molecule many times and count how many times each fragment appears, and then (ii) convert these counts into the desired distribution, based on statistical techniques (see SI section 3.1 for details). The underlying logic is that if a fragment duplicates many times in the original molecule, there would be many ways to fragment the original molecule so that this fragment appears, resulting in a large value in the distribution. 


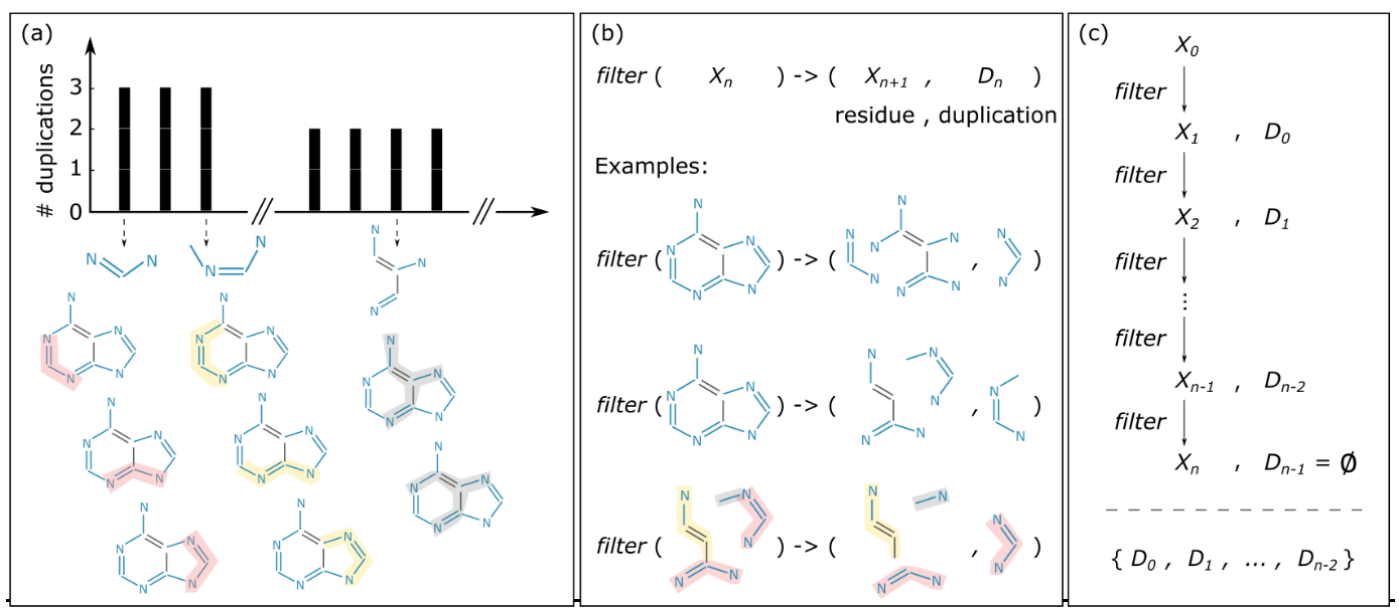

Fig.3. The general scheme of the Monte Carlo algorithm to compute the shortest assembly pathways of a molecule. (a) Schematic diagram of the fragments distribution that is obtained in the first step of the Monte Carlo algorithm. As we see, the first fragment has y-value 3, so it duplicates 3 times in adenine. The three adenines with colored patches under this fragment show where to find these three duplications. (b) The expected results of the operator filter(). Take the last panel as an example. In this case, this set of fragments is covered up by the drawn fragments highlighted in colors. The two red fragments are identical (thus duplicated), so one of them is filtered out as $D_{\mathrm{n}}$, and all the left-over fragments constitute the residue. (c) The scheme of the overall process to obtain one assembly pathway, shown at the bottom. For each time we run this process, we obtain one pathway. Run this process for a certain number of times (a predefined parameter), and the shortest assembly pathways among them are the final results.

Second, we introduce an operator filter $\left(X_{0}\right)$ where $X_{0}$ is a molecule, a fragment, or a set of fragments, which is used to find one duplicated fragment (if any) in $X_{0}$ and filter that out. This operator returns two results simultaneously: one is the duplicated fragment $D_{0}$ (if there is none, returns empty $\emptyset$ ) while the other is all the left-over parts of $X_{0}$ after $D_{0}$ is excluded which we call as the residue, $X_{1}$. So, we can denote $f i l t e r\left(X_{0}\right)->\left(X_{1}, D_{0}\right)$ (see Fig. 3b). The operator filter $\left(X_{0}\right)$ searches for duplications by first randomly drawing fragments from the distribution (obtained in the first step) to cover parts of $X_{0}$ until $X_{0}$ is completely covered by fragments, and then filtering the duplicated fragment $\left(D_{0}\right)$, leaving us the residue $X_{1}$ (if the fragments tried to cover up $X_{0}$ have no duplications, the operator will start over until there is one). Note that filter() involves a random process (i.e., randomly drawing fragments from the distribution), so for each run it may generate different results.

Now, we apply filter() to the original molecule $X_{0}$, and get $\left(X_{1}, D_{0}\right)$. Next, we apply filter() to the newly calculated residue $X_{1}$ and get $\left(X_{2}, D_{1}\right)$, and further apply filter() to $X_{2}$. Keep 
applying filter() to the newly calculated residue again and again until no duplication can be found, i.e., filter() returns an empty duplication $\emptyset$. Then, all the duplications obtained along the process constitute one assembly pathway (see Fig. 3c), whose assembly index can be easily calculated. So, for each time we run this process, we obtain one pathway. Finally, we repeat this process for a certain number of steps (a predefined parameter), the shortest assembly pathways obtained are the final results. The assembly index of the shortest pathway therefore determines the MA for the molecular target. See SI section 3 for the flowchart and details of the Monte Carlo algorithm (we also showed results of this algorithm for several other molecules). So far, this Monte Carlo algorithm deals with a single molecule, but can extend it to compute the shortest assembly pathways for a group of molecules, namely, the molecular assembly tree (see the definition and the slight extension in the next section).

\section{Molecular Assembly Trees}

The concept of assembly pathways and spaces can be naturally applied to two molecules, which allows us to look at the shortest assembly pathways that construct both simultaneously. If we consider molecule A and molecule B as one single "molecule X" with the disconnected part A and B, molecular assembly theory can be directly applied to molecule X. In general, the shortest assembly pathway of $\mathrm{X}$ is not the union of the shortest assembly pathway of $\mathrm{A}$ and that of B. As an example, consider adenine and another nucleobase thymine (Fig. 4a). The shortest pathway of adenine alone is indicated by the blue dashed arrows on the left whose MA is 7 (the same pathway as in Fig. 2a). The shortest pathway of thymine alone is indicated by the red dashed arrows on the right, which can also be written in multiset representation as $\{[7]\}$. Its MA is thus $(9-1)-(3-1)=6$ where 9 is the number of bonds of thymine and 3 is the number of bonds of building block [7]. 


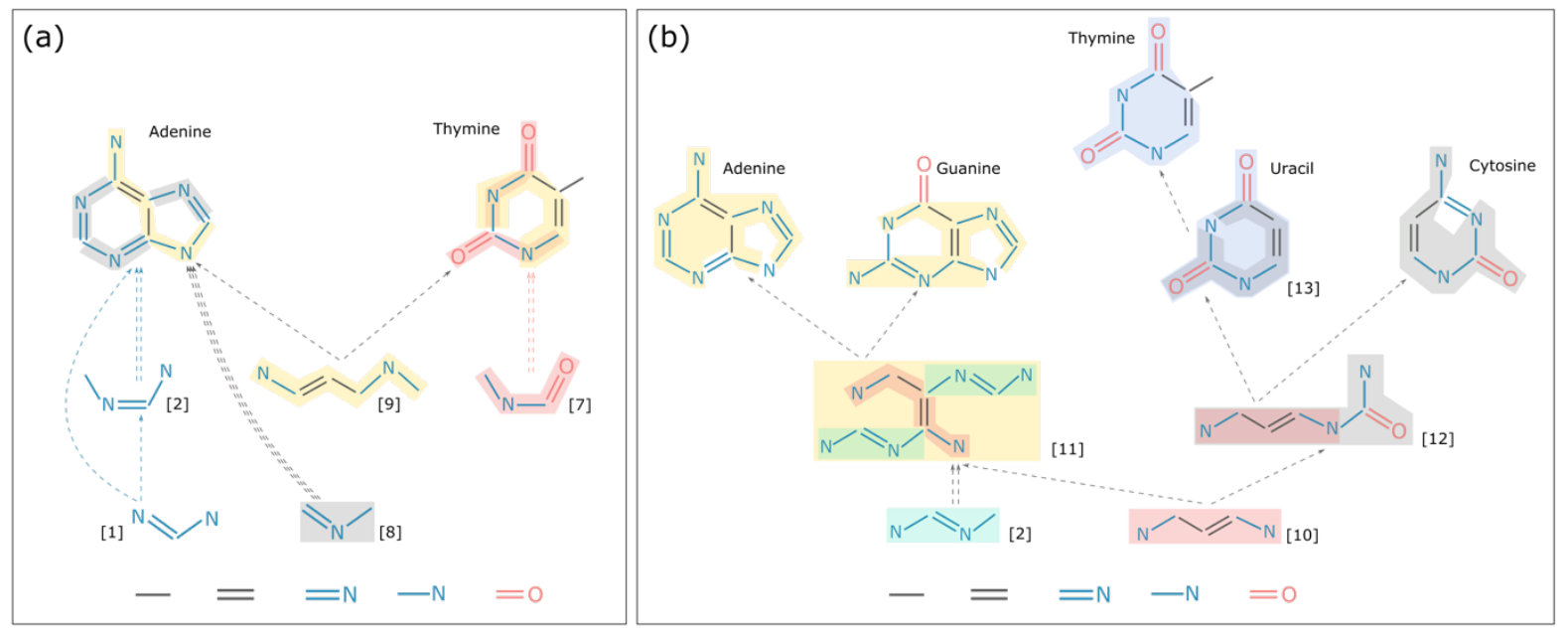

Fig. 4. (a) Assembly spaces of two molecules, adenine and thymine. The shortest assembly pathway for adenine alone is indicated by the blue dashed arrows, while the shortest assembly pathway for thymine alone is indicated by the red dashed arrows. The shortest assembly pathway to make adenine and thymine altogether is the one indicated by the black dashed arrows. (b) A molecular assembly tree for A, G, T, U and C, which can also be written as \{ [2], [10], [11], [12], [13] \}, whose index is $(11-1)+(12-1)+(9-1)+(8-1)+(8-1)-(3-1)-(4-1)-(10-1)-(7-1)-(8-1)=16$ where the non-one integers in the equation are the number of bonds of A, G, T, U, C, [2], [10], [11], [12] and [13] in turn. Note that, in both (a) and (b), the colored patches are just used to help the reader recognize the building blocks, and the color schemes are independent; and we also omitted the arrows starting from the basic building blocks, namely individual bonds, for a better visualization.

However, the shortest assembly pathway to make adenine and thymine altogether is indicated by the black dashed arrows in the middle, which does not overlap with either of the shortest pathways. It is $\left\{[8]^{2},[9]\right\}$ in multiset representation. Its pathway index is $(11-1)+(9-1)-1 * 2-$ $(5-1)=12$, which is smaller than $7+6$, the sum of the two individual MA's. It is straightforward to see that molecular assembly theory can be extended further to three or more molecules, which allows us to look at the shortest assembly pathways to construct a group of molecules. As we already see branches in the assembly space shown in Fig. 4a (e.g., building block [9] links two ends), the multi-molecular assembly spaces tend to have a treelike structure where different branches lead to different molecules, but the number of key building blocks is still relatively small. Therefore, we refer to the shortest assembly pathways to make a group of molecules altogether as the molecular assembly tree (assembly tree for short) of a group of molecules and refer to its index as this assembly tree's MA. An assembly tree shows the hierarchical relationships among chemical structures, fragments and molecules. It also reflects the idealistic shortest assembly pathways to make these involved molecules 
altogether, and thus contains their intrinsic structural information in the most compressed way.

Now, we build an assembly tree for the five nucleobases: adenine (A), guanine $(G)$, thymine (T), uracil (U) and cytosine(C), as an example to start with. We first extend the Monte Carlo algorithm from a single molecule to a group of molecules. In fact, we only need to treat this group of molecules as a single molecule with disconnected parts (it is the same object codewise, see details in SI section 4.1). We use this extended algorithm to compute the assembly tree, and then visualize the tree manually, as shown in Fig. $4 \mathrm{~b}$ (see SI section 4.3 for how to visualize the assembly tree based on the outputs of the algorithm). The tree can also be written in multiset representation: $\{[2],[10],[11],[12],[13]\}$; and its MA is 16 . What is intriguing is that, if for each step one bond is added, then 43 steps are needed to construct all of them (as A has 11 bonds and thus 10 steps, G has 12 bonds and thus 11 steps, T has 9 bonds and thus 8 steps, $\mathrm{U}$ has 8 bonds and thus 7 steps and $\mathrm{C}$ has 8 bonds and thus 7 steps), and the minimum number of steps to construct them separately using an assembly process is 33 (with A having an MA of 7, G having an MA of 8, T having an MA of 6, U having an MA of 5 and $\mathrm{C}$ having an MA of 7); while the shortest pathway to construct them altogether only requires 16 steps. This relatively low number reflects the fact that they share lots of common structures, and even the common structures share quite a few common substructures, resulting in the hierarchy as shown by the assembly tree. The assembly tree of nucleobases represents a highly related subset of chemical space.

The fact that the five nucleobases are closely related in the assembly tree indicates that it is not arbitrary or a frozen event that they serve as the fundamental units of the genetic code across all life on Earth, and that molecules may have been incorporated into proto-biological 
systems because of their relatedness. To test this idea, we built assembly trees for alternated nucleobases and showed their MA's. First, we selected ten random molecules that have the same formula as adenine $\mathrm{C}_{5} \mathrm{H}_{5} \mathrm{~N}_{5}$ (these random molecules were generated via MOLGEN 5.0, see SI section 5 for details), and then built ten assembly trees for each of these alternated adenines and the rest nucleobases G, T, C, U. We show MA's of these ten assembly trees at the bottom of Fig. 5 (as a histogram). We also show MA's of the assembly trees with alternated T, G, C, U, or all nucleobases alternated, to see how the tree compared to other possible ones in the chemical space. We can see that if one nucleobase is alternated, the assembly tree's MA is slightly larger than the real MA which is 16 as mentioned above, because the rest of the nucleobases still have quite a few common structures; while if all nucleobases are alternated, the assembly tree's MA is much larger, because few structures are shared. Therefore, if nature randomly "selects" nucleobases (with similar size as AGTCU) as the fundamental units of genetic code, MA of their assembly tree should be around 35 (Fig. 5). However, the real MA is 16 . These results demonstrate that the extant nucleobases have been selected from a relatively small subset of the chemical space even with the molecular formulas fixed, indicating that biologically relevant molecules are not arbitrary but a consequence of natural evolution for millions of years. 


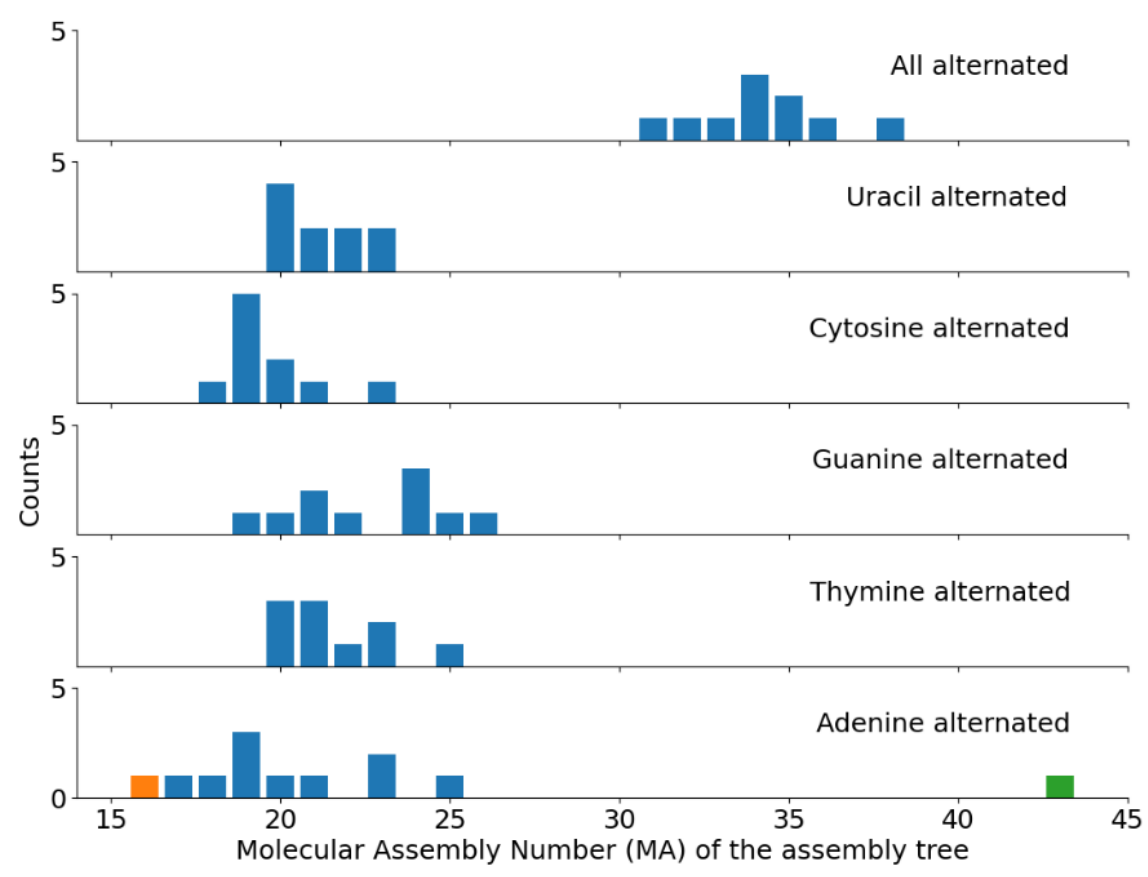

Fig. 5. Molecular assembly number (MA) of the assembly trees of alternated nucleobases. The top figure shows MA's of ten assembly trees where A, T, G, C and U are all alternated. It is a histogram, so the value on y-axis represents how many trees have such MA. In the 2nd, 3rd, 4th, 5th and 6th figure, only one nucleobase is alternated and all other nucleobases are the original. The orange bar at the left end is MA for the tree of real nucleobases; while the green bar at the right end is the assembly index of the longest assembly pathway to construct all of the real nucleobases. The green bar is located at 43 because if for each assembly step only one bond is added, 43 steps are then needed to construct all of the five real nucleobases (which is evidently the longest).

\section{Applications to Families of Molecules}

To demonstrate the power of assembly theory and spaces we employed assembly trees to study four distinct systems: biomolecules, gene sequences, the family of opiates and plasticizers. The aim is to show how the information carried by assembly trees is compressed and can help us explore chemical / sequence space in the context of the examples we have chosen. (i) By showing the assembly tree of a dozen vital biomolecules, it is possible to see that the assembly space that can be used to assemble many related molecules can effectively reduce the amount of information needed to generate the family of molecules. This has implications for the emergence of life, especially using assembly spaces to define the minimum set of molecules required to initiate evolution. (ii) When considering gene sequences, it is possible to use assembly spaces to generate the minimum genetic data that can be spliced together in an assembly space to build multiple genes within the minimum 
number of operations, again effectively reducing or compressing the information required to construct the given network of genes and reducing sequence duplication. (iii) By analyzing ten opiates, it can be seen that the chemical space constrained by the assembly tree is much smaller than those constrained by other information (e.g., chemical formula and molecular weight). This is important since the space of possible drug-like opiates is virtually infinite the assembly approach suggests how it is possible to focus on the compounds most likely to be important to biology which can exploit the assembly tree of opiates. If our hypothesis is correct, that is the assembly tree encodes structural information about the possible structures accessible with current biochemistry, then it is possible to use the assembly tree approach to map the important contingent information relevant to the opiate structures and evolved function. By using the opiate-assembly-tree, it will be possible to search and generate molecules that are intrinsically more similar, accessible, and potentially functional. (iv) Lastly, by showing the assembly tree of ten commonly-used plasticizers, it is possible to see how the information carried by the tree can help identify what molecules or chemical fragments are likely to be detected in the wider environment helping us understand and mitigate the effects of pollution from such compounds.

\subsection{Biomolecules}

For this study we picked a dozen vital biomolecules to construct their assembly tree, including the five nucleobases (A, G, T, C and U), pyruvate (a key intermediate in metabolic pathways across various organisms (44)), citrate (an intermediate of the vital metabolic pathways, the Krebs cycle, used by all aerobic organisms to release energy $(45,46))$. In addition, we included D-ribose (the carbohydrate that serves as the backbone of RNA amongst various other functions $(47,48)), \mathrm{NAD}+($ a vital cofactor that carries electrons from one reaction to another (49)), ADP, ATP and a symbolic RNA molecule. As we see in Fig. 6 
(together with Fig. 4), there are lots of structures are shared. We can imagine that as we include more biomolecules in the tree (e.g., various proteins and RNA, DNA sequences), more structures will be shared, and the tree will grow deeper and deeper but without growing much wider. This potentially "narrow" assembly tree is an indication that all of the vital biomolecules involved in extant life on Earth is not arbitrary but a consequence of millions of years of evolution.

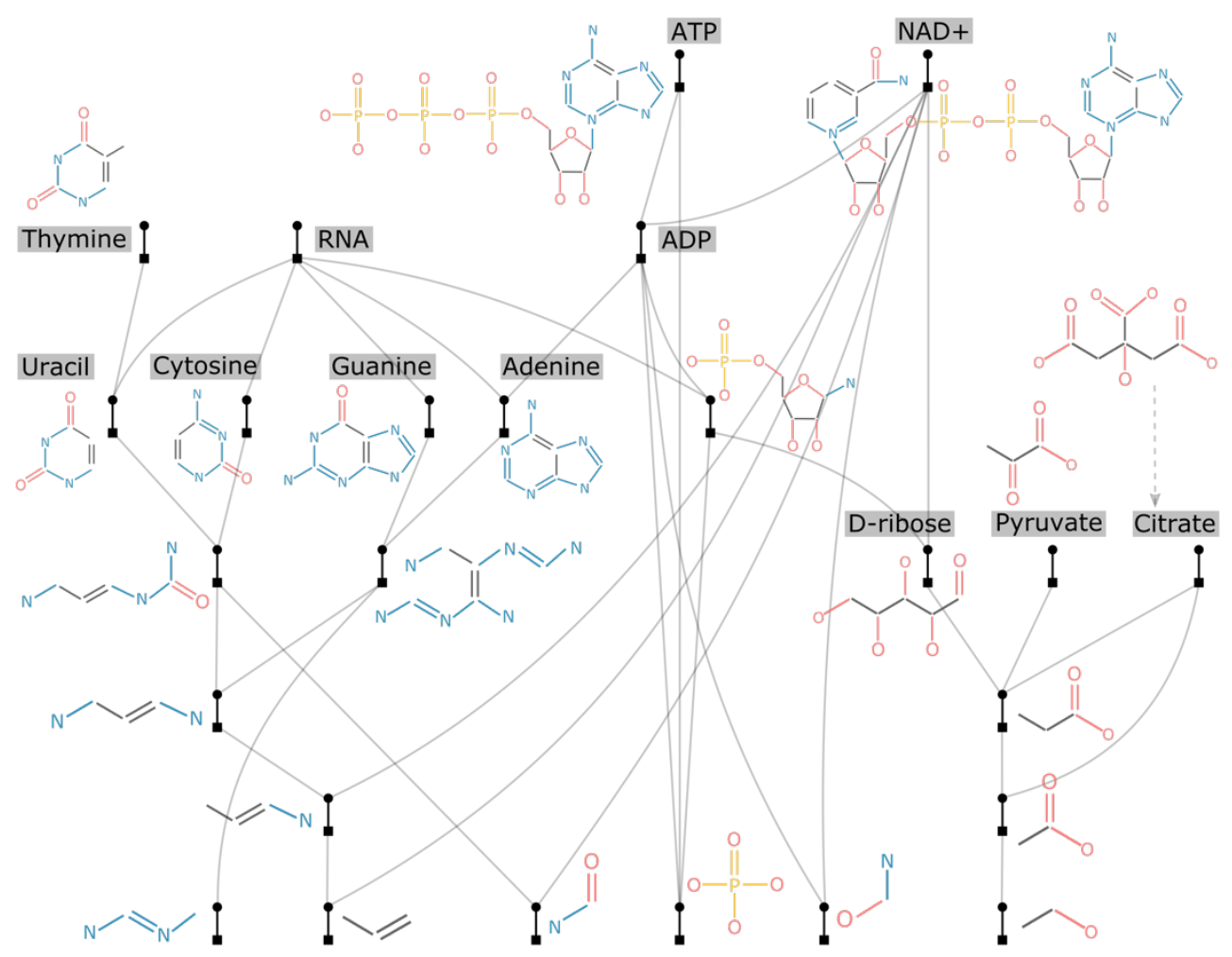

Fig. 6. The assembly tree of a dozen vital biomolecules, including the five nucleobases (A, G, T, C and U), pyruvate, citrate, D-ribose, NAD+, ADP, ATP and a symbolic RNA molecule. Note that all hydrogens are neglected.

In origin of life studies, one central problem is that most biomolecules (e.g., ATP, various enzymes, proteins, DNA sequences) are complex and thus seem very unlikely to emerge de novo from prebiotic chemistry, while life requires them altogether to function properly. This means it is not easy to imagine how life could simply emerge in the first place. An important clue we gain from this study is that lots of chemical structures are shared among these vital biomolecules. So, the difficulties to invent or produce them altogether will be much smaller than to invent or produce them de novo individually. In fact, the closer the biomolecules are 
in the assembly tree, the more effort can be saved and thus more likely for the emergence of life. We can hypothesize that this is also why the biomolecules exploited by extant life appear close in the assembly tree indeed else they would be too complex to emerge individually.

\subsection{Gene sequences}

So far, we have focused on constructing assembly trees for molecules, but assembly theory can be equally applied to other objects, including linear sequences of letters, such as gene sequences. We will take one hypothetical gene sequence $X$ (60 bases, Fig. 7$)$ as an example to illustrate how we can make use of the compressed information carried by $X^{\prime}$ s assembly tree to reconstruct itself with less efforts. The naïve method is to add one base at one time, and then 59 steps in total are needed to construct $X$. But notice that some segments are repeated for a few times. Therefore, if it is possible to produce these repetitive segments beforehand, it is possible to take them directly and combine them with other segments in a precise way to obtain $X$, and this requires less than 59 steps (as producing repetitive segments is relatively easy (50), e.g., polymerase chain reaction (PCR), the reconstruction of the original sequence takes less efforts than the naïve method). As the assembly tree is able to filter all of the repetitive and redundant information (i.e., it records the information of the sequence in the most compressed way), the information of this "precise way" is completely stored in the assembly tree.

Now we build $X^{\prime}$ 's assembly tree, as shown in Fig. 7 (as in this example we only deal with one sequence $X$, its assembly tree reduces to its shortest assembly pathway, but the same analysis applies to a group of gene sequences). Note that in this gene sequence case, we use nucleobases as the basic building blocks rather than chemical bonds as in the molecule cases. 


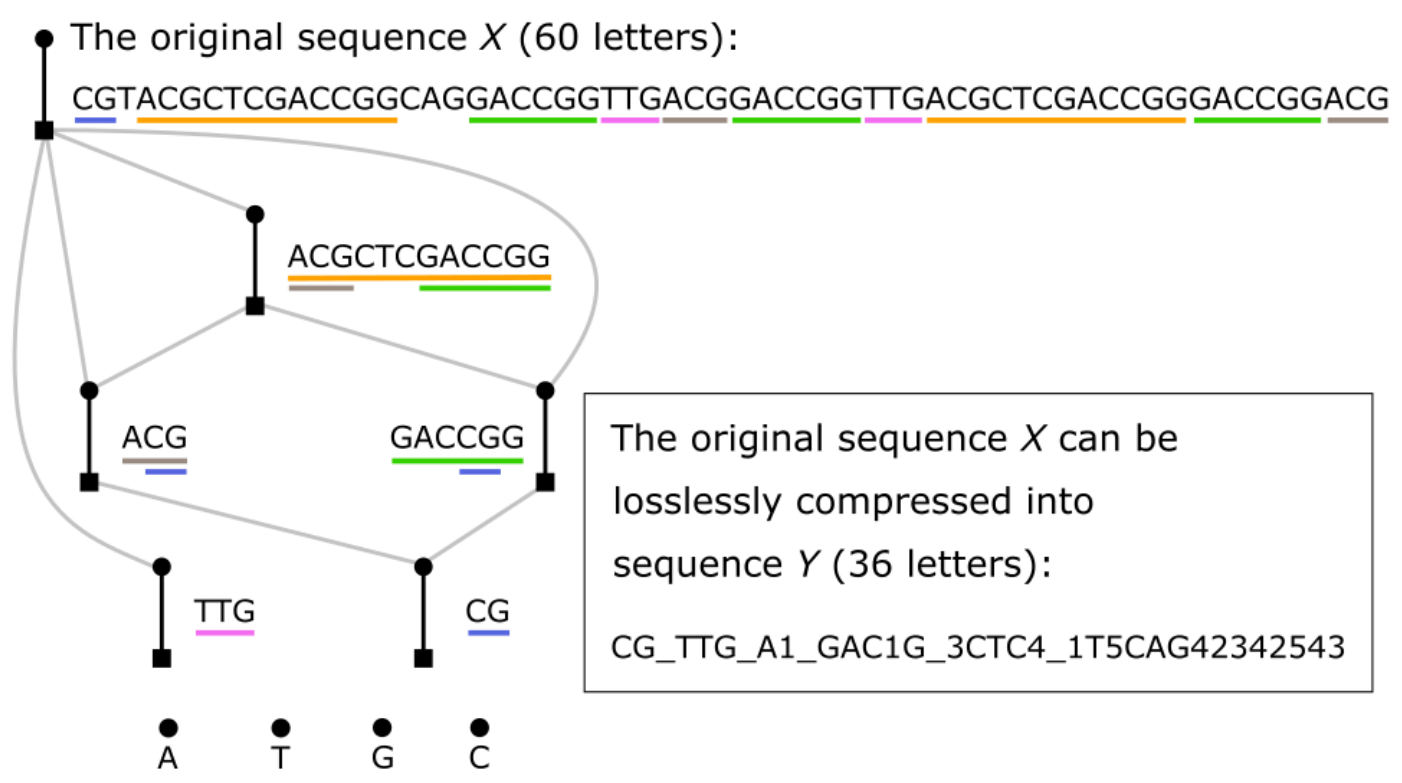

Fig. 7. The assembly tree of one hypothetical gene sequence $X$ (as here we only deal with one sequence $X$, it reduces to its shortest assembly pathway in the key-step representation). Here we use nucleobases as the basic building blocks rather than chemical bonds in the molecule cases. So we explicitly draw those nucleobases at the bottom for clarification.

This assembly tree can also be represented as a multiset $\left\{\mathrm{CG}^{2}, \mathrm{TTG}, \mathrm{ACG}^{2}, \mathrm{GACCG}^{3}\right.$, ACGCTCGACCGG\}, or equivalently, rewritten as a single sequence CG_TTG_A1_GAC1G_3CTC4_1T5CAG42342543, denoted as $Y$. We will then explain how to reconstruct $X$ based on $Y$, but first of all we will assume that any segment that has been constructed are available in any quantity afterwards, equivalent to assume that repetitive segments can be produced at no cost. Then, the first step to reconstruct $X$ is to construct $\mathrm{CG}$ by simply combining the individual bases $\mathrm{C}$ and $\mathrm{G}$ ( 1 step); and construct TTG by combining the individual bases T, T and G (2 steps). Secondly, construct A1 where "1" stands for the first segment in $Y$ which is CG. So, we only need 1 step to obtain ACG, since CG has been constructed before. Thirdly, construct GAC1G where we can reuse "1" again and we thus need 4 steps. Fourthly, construct 3 CTC4 where we can reuse " 3 " (A1, the 3 rd segment in $Y$ ) and "4" (GAC1G, the 4th segment in $Y)$, and we thus need 4 steps. Finally, we can construct the original sequence $X$ based on the last part of $Y$, namely "1T5CAG42342543" where the integer stands for the corresponding segment that has been obtained before and can thus be 
reused. So, we need 13 steps here. In total, we need $1+2+1+4+4+13=25$ steps which is much less than the naïve 59 steps.

Moreover, in order to quantify the increase of information from the original sequence $X(60$ letters) to the lossless and compressed version $Y$ (36 letters), we will employ Shannon entropy $(51,52)$, a widely-used quantity to describe the information of a string. Shannon entropy $H$ of a string $X$ (with $n$ letters) is defined as $H(X)=-\sum_{x} p(x) \cdot \log _{2} p(x)$, where $p(x)$ is the probability that the letter $x$ appears in this sequence $X$, which is set to be equal to the times $x$ appears in $X$ divided by $n$; and the sum goes through every distinct letter. Therefore, we obtained that Shannon entropy of the original sequence $H(X)$ is 1.851 , while $H(Y)$ is 3.251 , increased by 1.76 times.

\subsection{Opiates}

The search of opiate-based chemical space is important since it might allow us to be much more systematic in exploring where evolutionary systems will develop in future, since their future is contingent upon past structures and reaction cascades which are all ultimately encoded genetically. This means the search for new types of addiction-free pain medication, could be aided using the assembly tree approach. To illustrate how, we computed the assembly tree of ten compounds in the family of opiates (Fig. 8): some of them are found in the opium plant (morphine, codeine, thebaine and papaverine), some are synthetic opioids (fentanyl, remifentanil, methadone, pethidine, diamorphine, also known as heroin), and the last one is salvinorin A which is a kappa-opioid receptor agonist (53) found in the Salvia divinorum plant, although might not be properly considered as opioid itself. As we can see, the assembly tree captures some known features of the chemical space associated with these compounds: (i) Morphine, codeine, thebaine and diamorphine are clustered in one place, with 
a major structure shared. (ii) Fentanyl and remifentanil are close in the tree as they share a large structure. (iii) Methadone, pethidine and papaverine just have a relatively small benzyl group in common, and thus not so closely related, comparing to morphine and thebaine for example. (iv) Salvinorin A is distinct from others, as it only connects with other compounds via very small structures.

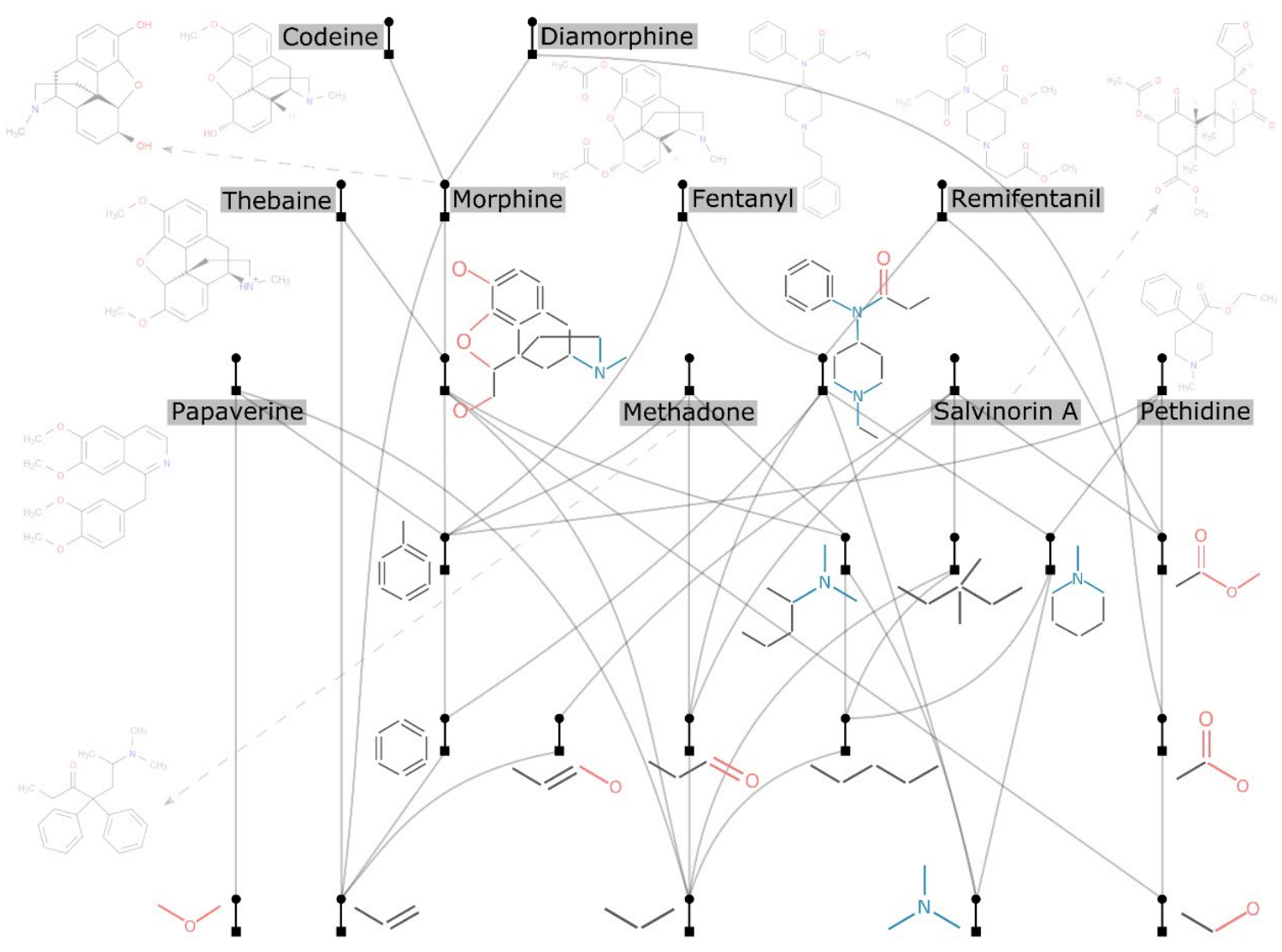

Fig. 8. The assembly tree of nine compounds in the family of opiates, and one kappa-opioid receptor agonist (salvinorin A). Some of these opiates are natural (morphine, codeine, thebaine and papaverine) while others are synthetic (fentanyl, remifentanil, methadone, pethidine and diamorphine, also known as heroin). For a clearer visualization, all opiates are made dimmer than other parts of the tree.

By computing the assembly tree, i.e., the shortest assembly pathways to construct these compounds simultaneously, we obtained not only the shortest, but also other longer assembly pathways (another output of our program, see SI section 6). The key building blocks included in all of these pathways---constituting the assembly space of these compounds---are highly related and encode their structural information. Comparing to the chemical space that includes these compounds and others with similar molecular weights, formulas, fingerprints, 
etc., which is normally used in drug discovery, this assembly space is much smaller. So, if we navigate, search, or manipulate the structures / molecules in this assembly space instead of the normal chemical space, the chance to discover new drug candidates could be much higher. Now the question is, how much smaller is the assembly space? As we employed the Monte Carlo (MC) method to compute the pathways, the size of the assembly space (namely the number of structures and fragments included) changes along with the number of MC steps. Nevertheless, we found that in this case the size of the assembly space is approximately one and half thousand, as we increase the number of MC steps up to the number that is far larger than the number needed to have very short pathways found (in this case, the smallest MA is found to be 105 while the total number of bonds of these ten opiates is 268). See details in SI section 6 for how the size of the assembly space changes with the number of MC steps. This small number is not because our approach or our program cannot find more unique structures, but the assembly space itself is intrinsically small since it only includes structures that are shared by at least two compounds, instead of any feasible structure or fragment. For comparison, we used MOLGEN 5.0 to calculate the total number of molecular structures possible using a total of 10 carbon, nitrogen, oxygen, or sulfur species, which amounts to over $10^{9}$ unique possibilities and is tremendously larger. Therefore, the navigation in the assembly space would be more focused and subtle.

\subsection{Plasticizers}

A vast part of the modern chemicals industry is the development and application of polymers which are used in packaging, structural materials, and have countless uses in modern devices. Plasticizers are added to polymers and formulations to make them more plastic, decrease viscosity, friction, and to increase flexibility (54). One issue with the compounds used as plasticizers is that they are molecular, and hence can be leached out of the polymer into the 
environment. This has potential problems since such compounds have been shown to be toxic, and have been shown to be potential endocrine disruptors (55). One big issue is that in general the evaluation of specific effects / prevalence of plasticizer molecules in the environment is hard as so many different types are in use, and many degradation pathways exist (55). This means that potentially vast numbers of molecules related to the plasticizer parent are present in the environment posing similar or even greater the health risks than the parent. However, by exploring the assembly tree of plasticizer molecules, it might be possible to map the molecules which are potentially contaminated, and even identify unknown or unexpected molecules.

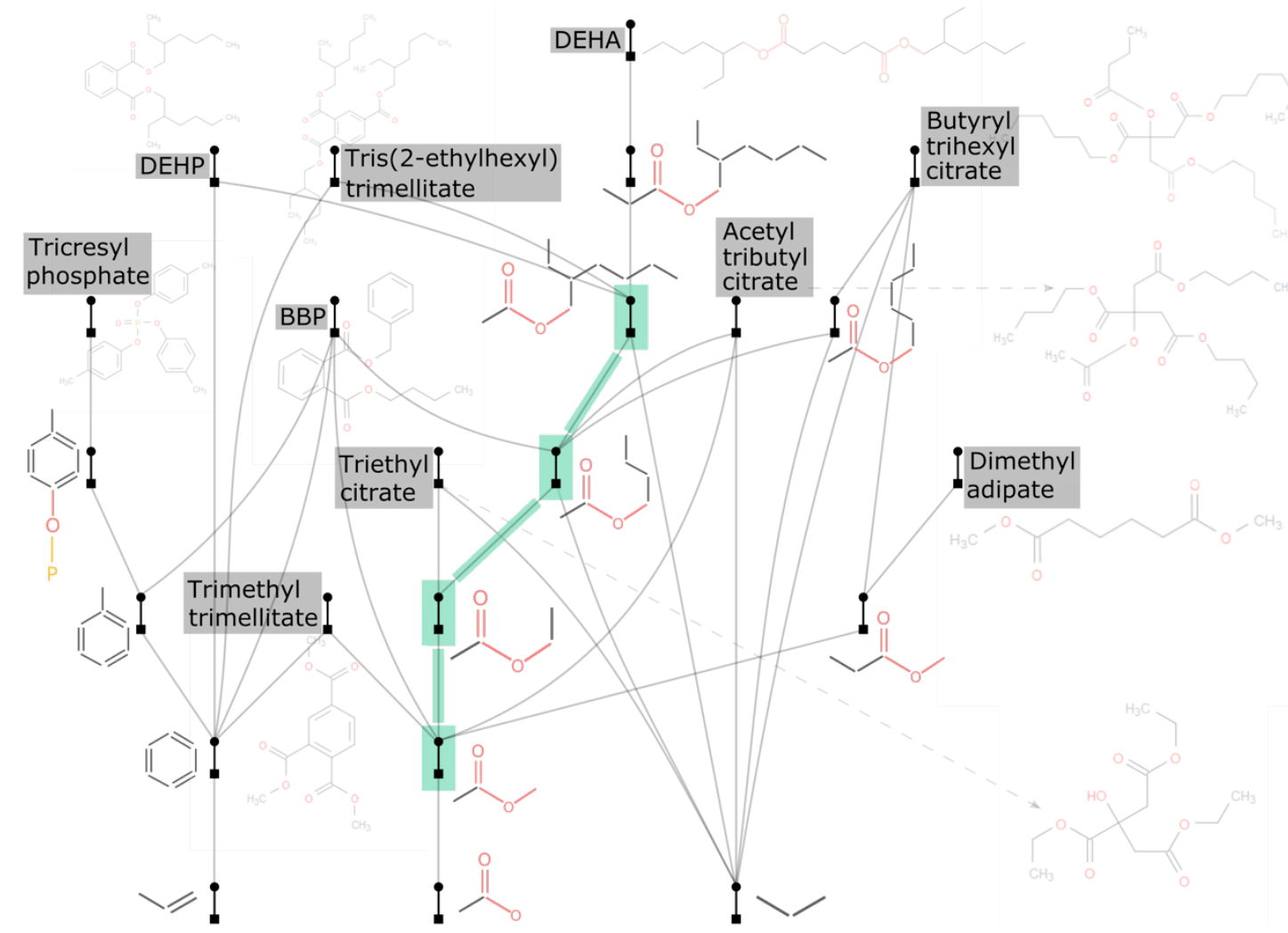

Fig. 9. The assembly tree of ten commonly-used plasticizers including BBP (Benzyl butyl phthalate), DEHP (di-2-ethylhexyl phthalate), DEHA (Bis(2-ethylhexyl) adipate) and others. For a clearer visualization, all plasticizers are made dimmer than other parts of the tree. The most central structures are highlighted green.

Here we built the assembly tree of ten commonly-used plasticizers including BBP (Benzyl butyl phthalate), DEHP (di-2-ethylhexyl phthalate), DEHA (Bis(2-ethylhexyl) adipate) and others. As seen from Fig. 9, these ten seemingly distinct molecules share lots of common 
structures (i.e., key assembly building blocks) which are also highly related. Generally speaking, the ten plasticizers which are not small molecules at all (most of them are in the range from 300 to $500 \mathrm{Da}$ ) are constructed by only a dozen of these key building blocks. In particular, the four central structures highlighted green directly or indirectly connect to most of them (except for tricresyl phosphate), and are highly related themselves. We can thus imagine, if we can identify these central chemical structures and fragments in an environment, we can be reasonably confident that this environment has been contaminated by these plasticizers, without trying to identifying each of them individually.

\section{Conclusions}

Our results demonstrate how Assembly Theory can be used to generate compressed representations of chemical space, while retaining the relevant chemical and structural information. We have shown how the concept of assembly spaces can be extended to multiple compounds and introduced notation to effectively represent the key features of such assembly spaces. A Monte Carlo algorithm for calculating the shortest assembly pathways and assembly spaces has been demonstrated and applied to molecules. Further, by developing an extended version, an assembly tree and an assembly space can be calculated for any collection of molecules. Using the developed algorithms, we constructed assembly trees that demonstrate how the structural similarities in the compounds constrain a chemical space.

We demonstrated this methodology in four distinct use cases, prebiotic chemistry, genetic engineering, drug discovery, and environmental chemistry. The assembly tree of biomolecules shows that those vital molecules used in biology represent a compressed subset of the possible compounds, suggesting they were subjected to evolutionary optimization rather than historical contingency alone. The analysis of gene sequences shown in Fig. 7 
demonstrates how assembly spaces can provide a lossless compression of sequences that retains the repeated motifs, demonstrating how complex gene sequences could be reconstructed from minimal genetic inputs. The analysis of the opioids shown in Fig. 8 provides a map to future drug development, by extracting the hierarchical relationships between compounds and identifying key components of possible drug candidates. Finally, by analyzing the assembly space of plasticizers, it was possible to identify structural motifs common to many different pollutants. These motifs can be used as general-purpose signals to identify entire classes of pollutants in complex environmental samples. These four different case studies demonstrate the wide applicability of assembly trees as a tool in a diverse set of disciplines. Finally, in future work we are going to develop an experimental approach to take a target set of molecules as templates for silico discovery and explore the enumeration of these targets using, random, fragment, and assembly-tree constrained approaches. The evaluation of both the properties and synthetic accessibility will provide a useful test of how assembly-trees can capture information encoded with a chemical network constructed using external constraints from either biochemistry or technology.

Methods Summary The algorithm described in this manuscript were implemented in $\mathrm{C}++$ can compiled using Visual Studio 2019. This code relied on the InChI standard libraries found at https://www.inchi-trust.org/. Details on the implementation and instructions on how to use the software can be found in the supplemental information.

Supplementary Information is linked to the online version of the paper on www.pnas.org/

Acknowledgements: The authors gratefully acknowledge financial support from the John Templeton Foundation (Grant 60025), EPSRC (Grant Nos EP/L023652/1, EP/R01308X/1, 
EP/J015156/1, EP/P00153X/1), the Breakthrough Prize Foundation and NASA (Agnostic Biosignatures award \#80NSSC18K1140), MINECO (project CTQ2017-87392-P) and ERC (project 670467 SMART-POM). We would like to acknowledge Alastair Murray for helpful discussions, Dr Liam Wilbraham for constructive comments on the initial manuscript, Dr Doug Moore and Dr Hessam Mehr for helpful discussions and input on the algorithmic implementations.

Author Contributions: LC conceived the theory and the hypothesis, designed the project, and coordinated the efforts of the research team. YL developed the algorithmic implementation of assembly theory and wrote the software to calculate assembly numbers and assembly spaces with help from SM. YL, CM and LC co-wrote the manuscript with input from all authors.

Competing Interests. The authors declare no competing interests.

\section{References}

1. C. M. Dobson, Chemical space and biology. Nature 432, 824-828 (2004).

2. C. Lipinski, A. Hopkins, Navigating chemical space for biology and medicine. Nature 432 , $855-861(2004)$.

3. R. S. Bohacek, C. McMartin, W. C. Guida, The art and practice of structure-based drug design: a molecular modeling perspective. Med Res Rev 16, 3-50 (1996).

4. P. Kirkpatrick, C. Ellis, Chemical space. Nature 432, 823-823 (2004).

5. S. Goto, Y. Okuno, M. Hattori, T. Nishioka, M. Kanehisa, LIGAND: database of chemical compounds and reactions in biological pathways. Nucleic Acids Res 30, 402-404 (2002). 
6. S. A. Sandford, M. Nuevo, P. P. Bera, T. J. Lee, Prebiotic Astrochemistry and the Formation of Molecules of Astrobiological Interest in Interstellar Clouds and Protostellar Disks. Chem Rev 120, 4616-4659 (2020).

7. R. J. Williams, The natural selection of the chemical elements. Cell Mol Life Sci 53, 816829 (1997).

8. H. J. Cleaves, C. Butch, P. B. Burger, J. Goodwin, M. Meringer, One Among Millions: The Chemical Space of Nucleic Acid-Like Molecules. Journal of Chemical Information and Modeling 59, 4266-4277 (2019).

9. J. Clardy, C. Walsh, Lessons from natural molecules. Nature 432, 829-837 (2004).

10. M. Frenkel-Pinter, M. Samanta, G. Ashkenasy, L. J. Leman, Prebiotic Peptides: Molecular Hubs in the Origin of Life. Chem Rev 120, 4707-4765 (2020).

11. Y. I. Wolf, N. V. Grishin, E. V. Koonin, Estimating the number of protein folds and families from complete genome data. J Mol Biol 299, 897-905 (2000).

12. A. M. Boldi, Libraries from natural product-like scaffolds. Curr Opin Chem Biol 8, 281$286(2004)$.

13. R. R. Breaker, Natural and engineered nucleic acids as tools to explore biology. Nature 432, 838-845 (2004).

14. D. Brohm et al., Natural products are biologically validated starting points in structural space for compound library development: solid-phase synthesis of dysidiolide-derived phosphatase inhibitors. Angew Chem Int Ed Engl 41, 307-311 (2002).

15. B. R. Stockwell, Exploring biology with small organic molecules. Nature 432, 846-854 (2004)

16. J. L. Reymond, The chemical space project. Acc Chem Res 48, 722-730 (2015). 
17. L. Ruddigkeit, R. van Deursen, L. C. Blum, J.-L. Reymond, Enumeration of 166 Billion Organic Small Molecules in the Chemical Universe Database GDB-17. Journal of Chemical Information and Modeling 52, 2864-2875 (2012).

18. C. Knox et al., DrugBank 3.0: a comprehensive resource for 'omics' research on drugs. Nucleic Acids Res 39, D1035-1041 (2011).

19. Y. Wang et al., PubChem: a public information system for analyzing bioactivities of small molecules. Nucleic Acids Res 37, W623-633 (2009).

20. J.-L. Reymond, L. Ruddigkeit, L. Blum, R. van Deursen, The enumeration of chemical space. Wiley Interdisciplinary Reviews: Computational Molecular Science 2, 717-733 (2012).

21. D. Rogers, M. Hahn, Extended-Connectivity Fingerprints. Journal of Chemical Information and Modeling 50, $742-754$ (2010).

22. P. Willett, Similarity-based virtual screening using 2D fingerprints. Drug Discov Today 11, 1046-1053 (2006).

23. J. Rosen, J. Gottfries, S. Muresan, A. Backlund, T. I. Oprea, Novel chemical space exploration via natural products. J Med Chem 52, 1953-1962 (2009).

24. R.-R. Griffiths, J. M. Hernández-Lobato, Constrained Bayesian optimization for automatic chemical design using variational autoencoders. Chemical Science 11, 577-586 (2020)

25. I. Y. Kanal, S. G. Owens, J. S. Bechtel, G. R. Hutchison, Efficient Computational Screening of Organic Polymer Photovoltaics. J Phys Chem Lett 4, 1613-1623 (2013).

26. N. M. O’Boyle, C. M. Campbell, G. R. Hutchison, Computational Design and Selection of Optimal Organic Photovoltaic Materials. The Journal of Physical Chemistry C 115, 16200-16210 (2011). 
27. C. Rupakheti, A. Virshup, W. Yang, D. N. Beratan, Strategy to discover diverse optimal molecules in the small molecule universe. J Chem Inf Model 55, 529-537 (2015).

28. A. M. Virshup, J. Contreras-Garcia, P. Wipf, W. Yang, D. N. Beratan, Stochastic voyages into uncharted chemical space produce a representative library of all possible drug-like compounds. J Am Chem Soc 135, 7296-7303 (2013).

29. G. M. Keseru, T. Soos, C. O. Kappe, Anthropogenic reaction parameters--the missing link between chemical intuition and the available chemical space. Chem Soc Rev 43, 5387 5399 (2014).

30. M. Awale, R. Visini, D. Probst, J. Arus-Pous, J. L. Reymond, Chemical Space: Big Data Challenge for Molecular Diversity. Chimia (Aarau) 71, 661-666 (2017).

31. T. Hoffmann, M. Gastreich, The next level in chemical space navigation: going far beyond enumerable compound libraries. Drug Discov Today 24, 1148-1156 (2019).

32. R. Gomez-Bombarelli et al., Automatic Chemical Design Using a Data-Driven Continuous Representation of Molecules. ACS Cent Sci 4, 268-276 (2018).

33. D. Duvenaud et al. (2015) Convolutional networks on graphs for learning molecular fingerprints. in Proceedings of the 28th International Conference on Neural Information Processing Systems - Volume 2 (MIT Press, Montreal, Canada), pp 2224-2232.

34. M. Ilardo, M. Meringer, S. Freeland, B. Rasulev, H. J. Cleaves, 2nd, Extraordinarily adaptive properties of the genetically encoded amino acids. Sci Rep 5, 9414 (2015).

35. S. M. Marshall, A. R. G. Murray, L. Cronin, A probabilistic framework for identifying biosignatures using Pathway Complexity. Philos Trans A Math Phys Eng Sci 375 (2017).

36. S. Marshall et al., Identifying Molecules as Biosignatures with Assembly Theory and Mass Spectrometry (2020), 10.26434/chemrxiv.13227881.v1.

37. D. Singh, A. M. Ibrahim, T. Yohanna, J. Singh, An overview of the applications of multisets. Novi Sad journal of Mathematics 37, $73-92$ (2007). 
38. P. Downey, B. Leong, R. Sethi, Computing Sequences with Addition Chains. SIAM Journal on Computing 10, 638-646 (1981).

39. D. E. Knuth, "Seminumerical Algorithms" in The Art of Computer Programming. (1997), vol. 2, pp. 461-485.

40. S. M. Marshall, D. Moore, A. R. G. Murray, S. I. Walker, L. Cronin, Quantifying the pathways to life using assembly spaces (2019), arXiv:1907.04649v2.

41. S. A. Cook (1971) The complexity of theorem-proving procedures. in Proceedings of the third annual ACM symposium on Theory of computing (Association for Computing Machinery, Shaker Heights, Ohio, USA), pp 151-158.

42. J. v. Leeuwen, Handbook of Theoretical Computer Science, Volumn A: Algorithms and Complexity (Elsevier, Amsterdam, 1998).

43. D. P. Kroese, T. Brereton, T. Taimre, Z. I. Botev, Why the Monte Carlo method is so important today. WIREs Computational Statistics 6, 386-392 (2014).

44. D. L. Nelson, A. L. Lehninger, M. M. Cox, "Lehninger principles of biochemistry". (W. H. Freeman, New York, 2008).

45. L. Nick, Life Ascending: The Ten Great Inventions of Evolution (W. W. Norton \& Co., New York, 2009).

46. E. Meléndez-Hevia, T. G. Waddell, M. Cascante, The puzzle of the Krebs citric acid cycle: assembling the pieces of chemically feasible reactions, and opportunism in the design of metabolic pathways during evolution. J Mol Evol 43, 293-303 (1996).

47. Y. Furukawa et al., Extraterrestrial ribose and other sugars in primitive meteorites. Proc Natl Acad Sci U S A 116, 24440-24445 (2019).

48. D. L. Tumbula, Q. Teng, M. G. Bartlett, W. B. Whitman, Ribose biosynthesis and evidence for an alternative first step in the common aromatic amino acid pathway in Methanococcus maripaludis. Journal of Bacteriology 179, 6010-6013 (1997). 
49. S.-J. Lin, L. Guarente, Nicotinamide adenine dinucleotide, a metabolic regulator of transcription, longevity and disease. Current Opinion in Cell Biology 15, 241-246 (2003).

50. A. D. Haimovich, P. Muir, F. J. Isaacs, Genomes by design. Nat Rev Genet 16, 501-516 (2015)

51. C. E. Shannon, A Mathematical Theory of Communication. Bell System Technical Journal 27, 379-423 (1948).

52. R. M. Gray, Entropy and Information Theory (Springer, Boston, MA, 2011).

53. B. L. Roth et al., Salvinorin A: a potent naturally occurring nonnitrogenous kappa opioid selective agonist. Proc Natl Acad Sci U S A 99, 11934-11939 (2002).

54. P. Walters, D. F. Cadogan, C. J. Howick, "Plasticizers" in Ullmann's Encyclopedia of Industrial Chemistry. (2020), 10.1002/14356007.a20_439.pub2.

55. R. U. Halden, Plastics and health risks. Annu Rev Public Health 31, 179-194 (2010). 
Supplementary Information for:

\section{Mapping the Molecular Tree of Life using Assembly Spaces}

Yu Liu, Cole Mathis, Stuart Marshall, Leroy Cronin

School of Chemistry, the University of Glasgow, University Avenue, Glasgow G12 8QQ, UK.

Email:lee.cronin@glasgow.ac.uk 


\section{Contents}

1 Glossary for Molecular Assembly Theory ................................................................

2 Why finding the shortest assembly pathway is at least as hard as NP-complete? .............4

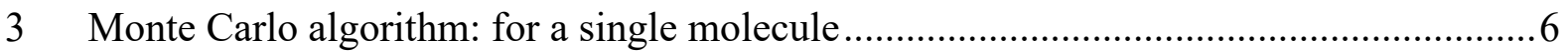

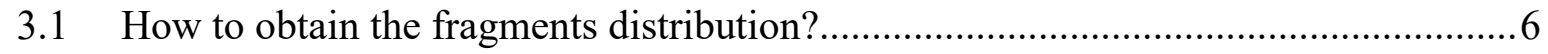

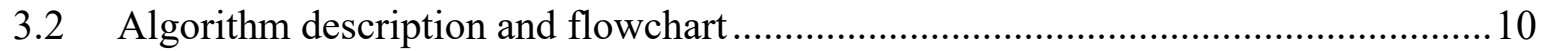

3.3 Executable program manual (AssemblyMC.exe) ..................................................... 15

3.4 Examples: several molecules' shortest assembly pathways calculated ....................20

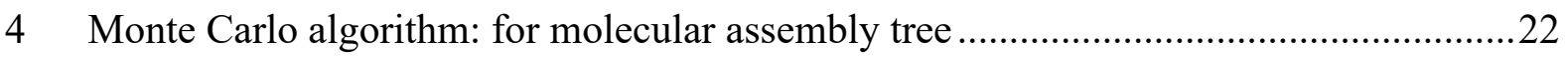

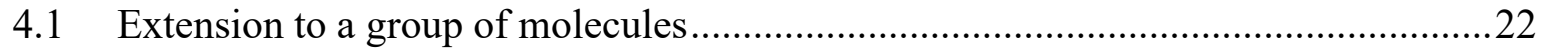

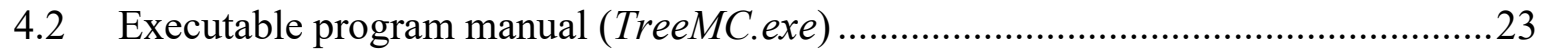

4.3 Visualize molecular assembly tree (based on multiset representation) .....................30

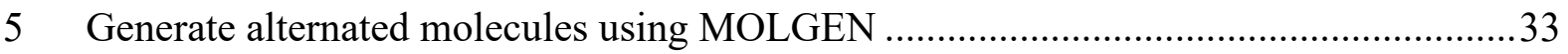

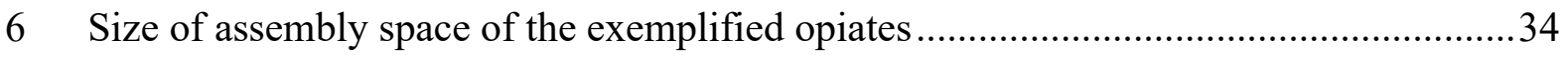

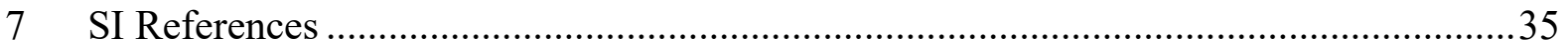




\section{Glossary for Molecular Assembly Theory}

- Molecular assembly theory: The name of the theory in general.

- (Assembly) building blocks: The units that are used to construct other units (e.g., in our molecular case, they are either chemical bonds, or chemical fragments, structures, molecules).

- The (Assembly) pool: The set of all assembly building blocks.

- One (assembly) step: It is defined as the three sequential operations (also mentioned in the main text): (1) Take two assembly building blocks from the assembly pool; (2) Join the two together in a way based on the particular system in question; (3) Put the new composite building block back into the assembly pool, and it will be considered as an assembly building block hereafter.

- One (assembly) pathway of a molecule: One sequence of assembly steps that successfully construct the target molecule.

- The (assembly) index of one assembly pathway: The number of assembly steps of the assembly pathway.

- The shortest (assembly) pathways of a molecule: The assembly pathways of the molecule that have the minimum number of assembly steps. Note that there could be more than one shortest assembly pathways.

- The molecular assembly number (MA) of a molecule: The assembly index of the shortest assembly pathway(s) of the molecule.

- The assembly space of one molecule: It is a term used in a more general sense, to refer to all of the assembly building blocks and the associated relationships included in many or all assembly pathways of the molecule. 
- Key (assembly) building blocks: The assembly building blocks that are used more than once in the assembly pathway. They describe the hierarchical relationships among the chemical fragments / structures / molecules.

- Key (assembly) steps: The corresponding assembly steps that make the key assembly building blocks.

- The key-step representation of an assembly pathway: It only displays key assembly building blocks in a hierarchical manner (see Fig. $2 \mathrm{a}$ in the main text).

- The multiset representation of an assembly pathway: It only displays key assembly building blocks, together with their multiplicities (namely, the number of times they occur), in the format of a multiset (see Fig. $2 d$ in the main text).

- The (Molecular) assembly tree of a group of molecules: It is defined as the shortest assembly pathways to make this group of molecules altogether. It can either be drawn in the key-step representation or written in the multiset representation.

- MA of an assembly tree refers to this assembly tree's assembly index.

\section{Why finding the shortest assembly pathway is at least as hard as NP- complete?}

As first introduced by Knuth (1), an addition chain for $n$ is defined to be a sequence of integers $1=a_{0}, a_{1}, a_{2}, \ldots, a_{r}=n$ with the property that $a_{i}=a_{j}+a_{k}$, for some $k \leq j<i$, for all $i=1,2, \ldots$, $r$. So, to find the shortest addition chain of an integer is a very special and simplified version of our question to find the shortest assembly pathway of a molecule (2), by simply considering an integer as a linear molecule made of carbons. As we know, to calculate the shortest addition chain for a given integer is a very difficult problem and no known algorithm can be guaranteed to finish in a reasonable time (although whether this problem is NP-complete or NP-hard has 
not been proven). On the other hand, a generalized version of the shortest addition chain problem---to find an addition chain that simultaneously forms each of a sequence of integers--has been proven to be NP-complete (3). This generalized version is exactly a largely simplified version of the question to compute the molecular assembly tree for a group of molecules (this concept is introduced in Section 4 of the main text).

In fact, to find the shortest assembly pathway for a single molecule is the combination of two problems: the first is to find the shortest addition chain of an integer, and the second is to determine if one graph (namely the graph representations of molecules, chemical structures and fragments) contains a subgraph that is isomorphic to another graph, which is an example of the subgraph isomorphism problem, which has been proven to be NP-complete (4). Besides, to compute the assembly tree for a group of molecules is the combination of (i) to find an addition chain that simultaneously forms each of a sequence of integers and (ii) the subgraph isomorphism problem. Therefore, either to find the shortest assembly pathway for a single molecule or to compute the assembly tree for a group of molecules is at least as hard as an NPcomplete problem. See the following Table where we have summarized the computational complexity of these problems.

\begin{tabular}{l|lll}
\hline Problems & $\begin{array}{l}\text { To find the shortest assembly } \\
\text { pathway for a single molecule }\end{array}$ & $\begin{array}{l}\text { To find the shortest addition } \\
\text { chain of an integer }\end{array}$ & $\begin{array}{l}\text { Subgraph } \\
\text { isomorphism } \\
\text { problem }\end{array}$ \\
\hline $\begin{array}{l}\text { Computational } \\
\text { complexity }\end{array}$ & At least as hard as NP-complete & $\begin{array}{l}\text { Hard, but not been proven to } \\
\text { be NP-complete or NP-hard }\end{array}$ & NP-complete \\
\hline \hline Problems & $\begin{array}{l}\text { To compute the assembly tree } \\
\text { for a group of molecules }\end{array}$ & $\begin{array}{l}\text { To find an addition chain that } \\
\text { simultaneously forms each } \\
\text { of a sequence of integers }\end{array}$ & $\begin{array}{l}\text { Subgraph } \\
\text { isomorphism } \\
\text { problem }\end{array}$ \\
\hline $\begin{array}{l}\text { Computational } \\
\text { complexity }\end{array}$ & At least as hard as NP-complete & NP-complete & NP-complete \\
\hline
\end{tabular}




\section{Monte Carlo algorithm: for a single molecule}

\subsection{How to obtain the fragments distribution?}

This part of the algorithm is to calculate a distribution that shows how many times a molecular structure or fragment is duplicated in the original molecule. For convenience, here we use a string (Fig. S1a) as an example to illustrate instead of a molecule, but the same principle applies to molecules. Now, the question is to find how many instances of each fragment (namely, substring) are contained in the original string. We see that the fragment CUCGAC appears twice, GAC appears four times, CG appears three times, etc. Ultimately, the algorithm should be able to tell us those information.

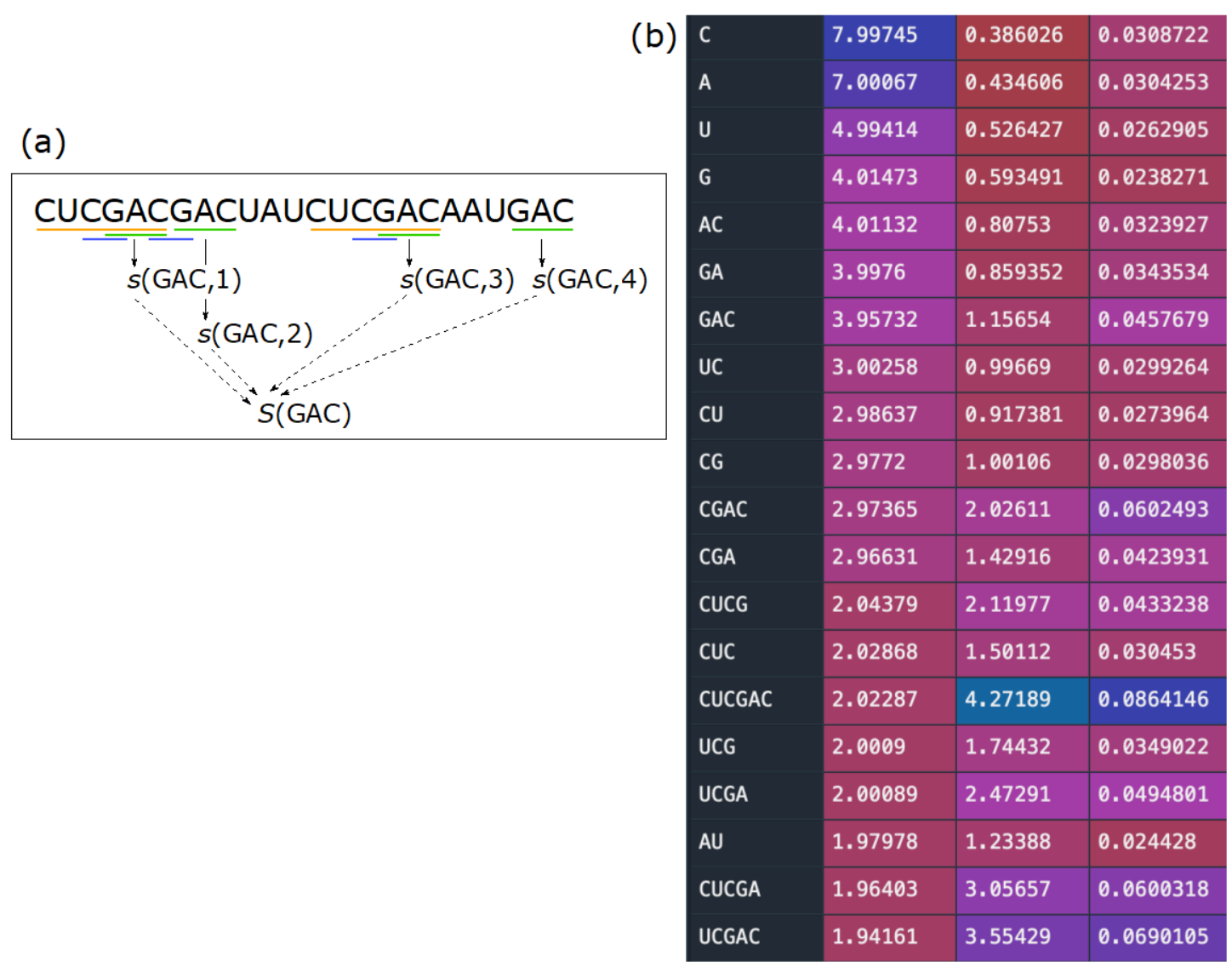

Fig. S1. (a) The exemplified original string. (b) The output of the algorithm. The first column shows how many substance of each fragment are contained in the original string. Because this algorithm is statistical, all the values are not integers, but close to. The third column shows the measurement error (standard deviation). The second column equal to the third divides the first column, in percentage. All fragments whose measurement error is larger than 5\% are dropped. 
More precisely, the algorithm should tell us the value $w(S)$ that describes how many instances of each fragment $S$ are contained in the original string. This algorithm is conceptually simple, and has two main steps:

1. Randomly fragment the original string for many times, and count how many times each fragment appears, thus a histogram obtained.

2. Weight the counts more for longer fragments. Generally speaking, the weighting scheme is: "the weighted counts of a fragment $S$ " is proportional to "(the actual counts of $S) \times(2$ to the power of the length of $S)$ ".

3. Normalized the weighted counts, and then we obtain $w(S)$.

The output of this algorithm applied to the exemplified string is shown in Fig. S1b. The details of why this can work are shown in the following.

- Notation

First of all, we denote a specific fragment as $s$ (lowercase). Note that if we want to distinguish two fragments that are identical but in different positions, we will use the lowercase $s$. For example, in Fig. S1a, the fragment GAC that consists of the 4th, 5th and 6th letter is denoted as $s(\mathrm{GAC}, 1)$; while we denote another GAC fragment that consists of the 7 th, 8 th and 9 th letter as $s(\mathrm{GAC}, 2)$. On the other hand, if we do not want to distinguish fragments that are identical but in different positions, we will use $S$ (uppercase). As shown in Fig. S1a, all fragments GAC are denoted as $S(\mathrm{GAC})$. Therefore, the ultimate goal of this algorithm is to find $w(S)$, rather than $w(s)$. With that being clear, we can proceed.

Theoretically, we have $n$ : the number of links of the original string (i.e., the number of letters minus 1); 
$m$ : the number of links of the fragment in question;

$N=2^{n}-1:$ the total number of unique fragmenting events;

$M_{a}$ and $M_{b}$ : in these $N$ unique events, for any fragment $s$ on the edge, it will appear for $M^{a}=$ $2^{n^{-m-1}}$ times, while for any fragment $s$ in the middle, it will appear for $M_{b}=2^{n^{-m}-2}$ times; $\Pi$ : the total number of fragments appeared in these $N$ unique events, that is

$$
\Pi=2\left(\begin{array}{l}
n \\
1
\end{array}\right)+3\left(\begin{array}{l}
n \\
2
\end{array}\right)+4\left(\begin{array}{l}
n \\
3
\end{array}\right)+\cdots+(n+1)\left(\begin{array}{l}
n \\
n
\end{array}\right)=(n+2) \cdot 2^{n-1}-1
$$

Empirically, we have

$\widehat{N}$ : the number of fragmenting events in the experiments (referred to as fragmenting trails);

$\widehat{\Pi}:$ the total number of fragments appeared in these $\widehat{N}$ empirical trails.

So, in these $\widehat{N}$ empirical trails, the number of times that one fragment $s$ appears is denoted as $\hat{h}_{x}(s)$. If $s$ is on the edge, it is denoted as $\hat{h}_{a}(s)$; if $s$ is in the middle, it is denoted as $\hat{h}_{b}(s)$. Therefore, based on Wilson score interval in statistics, the probability of the fragment $s$ appears is (mean \pm standard deviation):

$$
\hat{p}_{x}(s)=\frac{\hat{h}_{x}(s)+\frac{z^{2}}{2}}{\hat{\Pi}+z^{2}} \pm \frac{z}{\hat{\Pi}+z^{2}} \sqrt{\frac{\hat{h}_{x}(s)\left(\hat{\Pi}-\hat{h}_{x}(s)\right)}{\hat{\Pi}}+\frac{z^{2}}{4}}
$$

where $x$ is replaced by $a$ if the fragment $s$ is on the edge, and $x$ is replaced by $b$ if $s$ is in the middle; $z$ is the probit function (when the confidence level is $95 \%$, then $z=1.96$ ). One step further, the probability of the fragment $S$ appears on the edge is:

$$
\hat{p}_{a}(S) \doteq \frac{\hat{H}_{a}(S)+\frac{z^{2}}{2}}{\hat{\Pi}+z^{2}} \pm \frac{z}{\hat{\Pi}+z^{2}} \sqrt{\frac{\hat{H}_{a}(S)\left(\hat{\Pi}-\hat{H}_{a}(S)\right)}{\hat{\Pi}}+\frac{z^{2}}{4}}
$$

where $\widehat{H}_{a}(S)$ is the total number of times that $S$ appears on the edge. The probability of the fragment $S$ appears in the middle is:

$$
\hat{p}_{b}(S) \doteq \frac{\hat{H}_{b}(S)+\frac{z^{2}}{2}}{\hat{\Pi}+z^{2}} \pm \frac{z}{\hat{\Pi}+z^{2}} \sqrt{\frac{\hat{H}_{b}(S)\left(\hat{\Pi}-\hat{H}_{b}(S)\right)}{\hat{\Pi}}+\frac{z^{2}}{4}}
$$


where $\widehat{H}_{b}(S)$ is the total number of times that $S$ appears in the middle.

- Now we can show how to calculate $w(S)$ based on the empirical data

Referring to the string in Fig. S1a, the fragment GAC has 4 instances. In the $N$ unique fragmenting events, the fragment $s(\mathrm{GAC}, 1)$ will appear $M_{b}=2^{23-2-2}$ times, denoted as $h_{b}(s(\mathrm{GAC}, 1))$. The same for $s(\mathrm{GAC}, 2)$ and $s(\mathrm{GAC}, 3)$. On the other hand, in the $N$ unique fragmenting events, the fragment $s(\mathrm{GAC}, 4)$ will appear $M^{a}=2^{23-2-1}$ times, denoted as $h_{a}(s(\mathrm{GAC}, 4))$. Eventually, we can use these counts $h_{x}$ to achieve the answer 4, i.e.,

$$
\begin{aligned}
w(S=G A C) & =\frac{h_{a}(s(G A C, 4))}{M_{a}}+\frac{h_{b}(s(G A C, 1))}{M_{b}}+\frac{h_{b}(s(G A C, 2))}{M_{b}}+\frac{h_{b}(s(G A C, 3))}{M_{b}} \\
& =\frac{h_{a}(s(G A C, 4))}{M_{a}}+\frac{h_{b}(s(G A C, 1))+h_{b}(s(G A C, 2))+h_{b}(s(G A C, 3))}{M_{b}}
\end{aligned}
$$

In general, we have

$$
\begin{aligned}
w(S) & =\frac{\Sigma_{i} h_{a}\left(s_{i}\right)}{M_{a}}+\frac{\Sigma_{j} h_{b}\left(s_{j}\right)}{M_{b}} \\
& =\frac{H_{a}(S)}{M_{a}}+\frac{H_{b}(S)}{M_{b}}
\end{aligned}
$$

where $H_{a}(S)$ is the total number of times that $S$ appears on the edge, and $H_{b}(S)$ is for $S$ in the middle. If we substitute the empirical values, we obtain the measured $w(S)$, i.e., $\widehat{w}(S)$. In order to evaluate the empirical error, we use Eq. (S3.1) and (S3.2) to calculate $H_{a}(S)$ and $H_{b}(S)$, rather than directly using $\widehat{H}_{a}(S)$ and $\widehat{H}_{b}(S)$. Therefore, we have

$$
\hat{w}(S)=\frac{\Pi \cdot \hat{p}_{a}(S)}{M_{a}}+\frac{\Pi \cdot \hat{p}_{b}(S)}{M_{b}}
$$

Equivalently, the mean is

$$
E[\hat{w}(S)]=\frac{\Pi}{M_{a}} \frac{\hat{H}_{a}(S)+\frac{z^{2}}{2}}{\hat{\Pi}+z^{2}}+\frac{\Pi}{M_{b}} \frac{\hat{H}_{b}(S)+\frac{z^{2}}{2}}{\hat{\Pi}+z^{2}}
$$

and the standard deviation is 


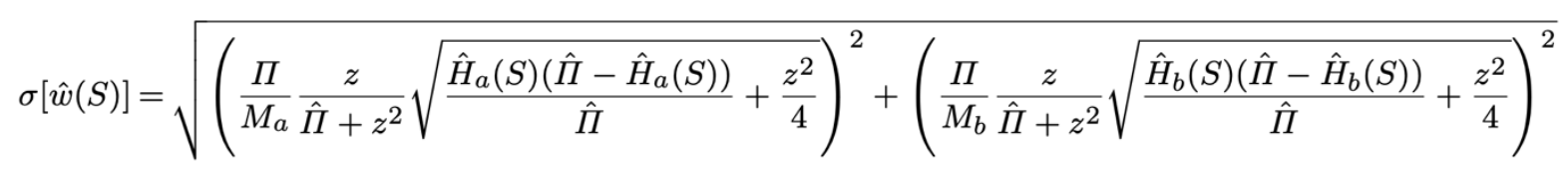

To summary, by randomly fragmenting the original string many times, we can get the empirical $\widehat{H}_{a}(S), \widehat{H}_{b}(S)$ and $\widehat{\Pi}$, and we can then calculate $E[\widehat{w}(S)]$ based on Eq. (S3.3), which is the empirical value of $w(S)$.

\subsection{Algorithm description and flowchart}
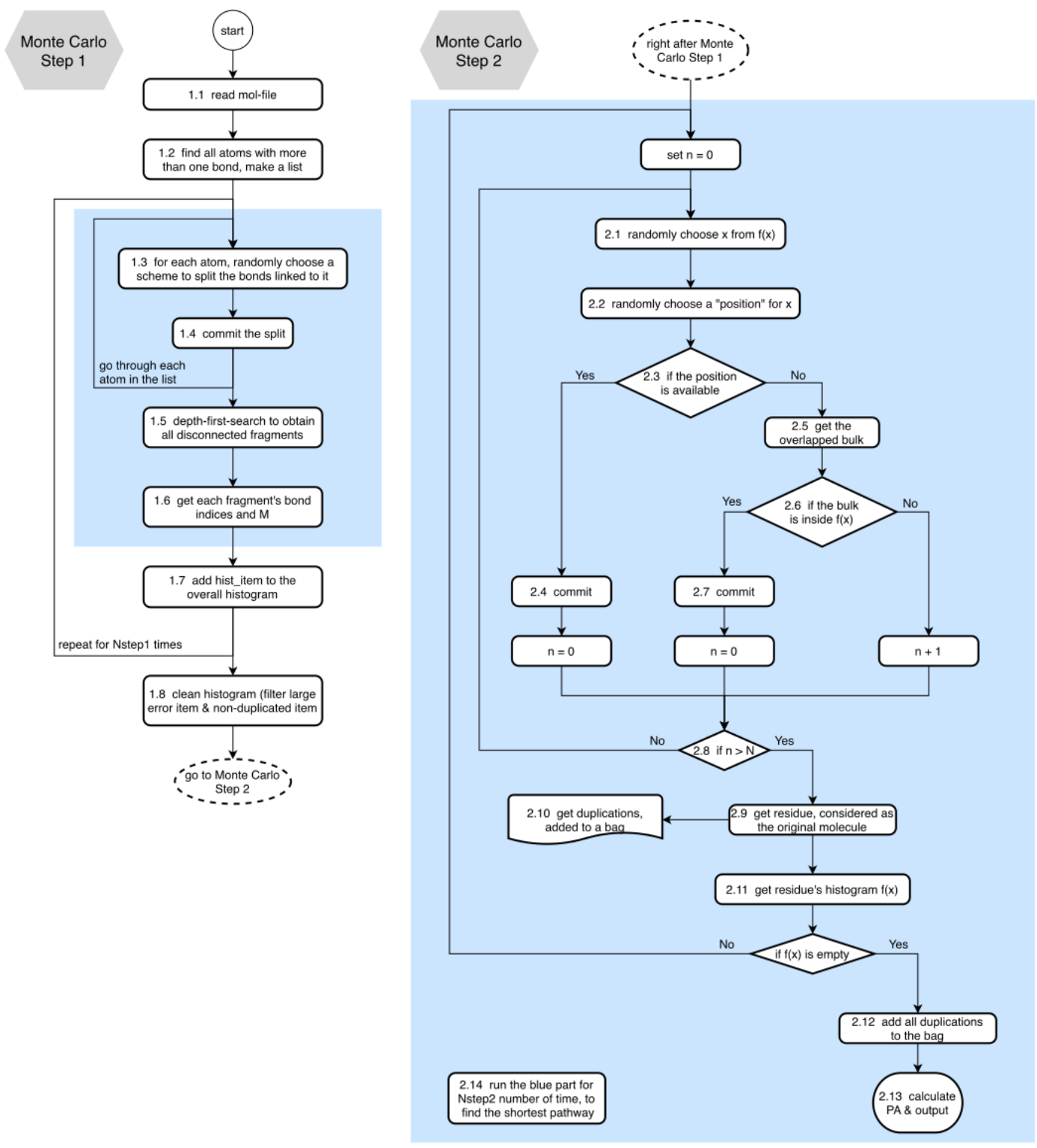

Fig. S2. The flowchart of the Monte Carlo algorithm to compute the shortest assembly pathways for a single molecule. The algorithm is mainly divided into two steps. 


\section{Monte Carlo Step 1:}

1.1. Read mol-file to construct a "molecule" object ( $M O L_{-} B O N D$ class), which stores all of the atoms and bonds, also giving each atom an index and each bond (connecting two atoms) an index.

1.2. Find all atoms that have more than one bond linked to it, and make a list recording them.

1.3. For each atom in the list, randomly choose one possible scheme to "split the bonds". For example, this atom has bond 1, 3 and 6 linked to it; so in total it has five ways to split the bond: no split (136), three ways to split into two $(1,36),(3,16)$ and $(6,13)$, split into three $(1,3,6)$. In general, there is a mathematical formula to generate all of the possible splitting.

1.4. Commit the split, meaning: add atoms to the split end of the bond. For example, if you choose to cut into $(1,36)$, then add an atom to the split end of bond 1 ; for bond 3 and 6 , we don't need to add atoms since we can just use the original end-atom.

1.5. After go through all atoms that have more than 1 bond linked to it, we obtain a molecule with bonds cut (still a $M O L_{-} B O N D$ class object). Now we can use depth-first-search algorithm to work out all the disconnected parts. Each disconnected part is a fragment of this molecule. Then we obtain a list of fragments.

1.6. For each fragment, we know which bonds it consists of, and we can also calculate the number M (referring to SI section 3.1 for details about M). All of these gives hist_item (the information of one item of the overall fragment histogram).

As 1.3 - 1.6 (the blue part in the flowchart Fig. S2) are the main processes in Step 1, we summarize them further here: the outcome of $1.3-1.6$ is to obtain hist_item that will be used in 1.7 to construct the distribution of fragments of this molecule (namely, a histogram). hist_item is a list of tuples each of which stores the complete information of a fragment (so this list of tuples represents all of the fragments that constitute this molecule). Each tuple consists of three things: InChI string of this fragment, the bond indices that constitute this fragment 
(called "fragment identifier"), a number (denoted as M) associated with this fragment that will be used to weight the counts to get the final histogram. Refer to SI section 3.1 to see how M is calculated and used to weight.

1.7. Add hist_item to the overall histogram, where the $\mathrm{x}$-axis is the InChI of a particular structure and y-axis is the count of that structure. A map is also constructed which associates a list of fragment identifiers and corresponding M values with each InChI string. That is, the key of this map stores InChI strings; and under any InChI, there is a list of fragment identifiers (that all have the same $\mathrm{InChI}$ ) and the corresponding $\mathrm{M}$.

After repeating 1.3 - 1.7 for Nstepl (a predefined parameter) number of times, we get the overall histogram. The y-axis is the count of how many times a fragment appears in Nstep1 random cuttings. For example, we are looking at this molecule $\mathrm{C}-\mathrm{N}-\mathrm{C}-\mathrm{O}-\mathrm{C}-\mathrm{N}-\mathrm{C}$; and the fragment $\mathrm{C}-\mathrm{N}-\mathrm{C}$ appears 1 million times in all these cuttings, then $\mathrm{y}$-axis for $\mathrm{C}-\mathrm{N}-\mathrm{C}$ is 1 million. But 1 million is not what we want. Instead, we want to know how many times a fragment has been duplicated in this molecule. In this case, it is 2 . So we need to convert the counts (1 million) to number of duplications (two). This conversion can be done by using the counts and the number M (see details in SI section 3.1), which gives the statistical measurement of how many times a fragment is duplicated, mean \pm error.

1.8. Clean up the histogram. We first delete the items that have the error $>$ Hist_Err_Threshold (a predefined parameter, $5 \%$ by default). Then we round the means to integers. Finally, we delete the items whose corresponding y-axis values are 1 (i.e., it only appears once in this molecule). 
Monte Carlo Step 1 is now complete. In the end, we obtain a histogram where $\mathrm{x}$-axis is the InChI string of fragment and y-axis is how many times it duplicates in this molecule (with $100 \%-5 \%=95 \%$ confidence by default).

\section{Monte Carlo Step 2:}

2.1. Denote the histogram obtain from step 1 as $f(x)$ where $x$ represent a fragment. Create a list $S$ recording all the bond indices of the original molecule, e.g., $(1,2,3,4,5,6,7,8,9,10,11,12)$, to represent which bonds have been taken (now it means all bonds are available). Randomly choose a fragment $x$ based on the histogram $f(x)$, i.e., the relative y-value represents the probability that a certain $x$ is chosen.

2.2. Randomly choose a "position" for $x$. Here "position" means the "fragment identifier". Recall that in step 1 we have obtained a map linking fragment's InChI string with a list of fragment identifiers (i.e., the bond indices that constitute the corresponding fragment). For example, if this chosen fragment has 3 duplications: one is $(1,2,9)$, one is $(3,5,6)$ and one is $(7,8,12)$; then here we randomly chose one, say, $(3,5,6)$.

2.3. Check if this position is available, that is, check if each element in $(3,5,6)$ is in the list $S$. Initially, $S=(1,2,3,4,5,6,7,8,9,10,11,12)$, so the answer would be yes. But if $S=$ $(1,5,7,8,9,12)$, then the answer would be no.

2.4. Commit, so we delete 3,5 and 6 from the list $S$, means that we consider this fragment has been taken.

2.5. If the position is not available, then it means $x$ must be overlapped with one or more already-taken fragments. In the flowchart, get the overlapped bulk means figuring out these already-taken fragments, say, $\left(y_{1}, y_{2}, y_{3}, \ldots\right)$, and make a new fragment that consists of $x$ and $y_{1}, y_{2}, y_{3} \ldots$ We denote this big new fragment as bulk $x$ '.

2.6. Check if $x^{\prime}$ is contained in the histogram. If yes, it means $x^{\prime}$ is a duplicated fragment; 
otherwise not.

2.7. Commit here means delete each index in $x$ ' from the list $S$, meaning they have been taken.

2.8. Here $n$ means for how many times we cannot find an available position for new fragments continuously. If it is larger than a predefined number $N$, we stop this searching process, meaning that we assume no position available. By default, $N$ is set to twice the number of bonds of the molecule.

2.9. We now have a list of fragments that are used to make the molecule. Now we check which fragments appear more than once in this list, the "duplications", and then remove them, storing them separately ( 2.10 below). The leftover fragments constitute the part we call the "residue".

2.10. Refer to 2.9 above.

2.11. The residue is a list of non-duplicated fragments. Now we consider them as one whole molecule (namely a $M O L \_B O N D$ object) although it consists of disconnected parts. We need to obtain a fragment histogram for it, similar to $f(x)$ described above to the original molecule. Naively we can repeat Monte Carlo Step 1 on the residue to get such a histogram, but we have a shortcut here: We check every InChI string in the original $f(x)$, to see whether it is a fragment of the residue. If it is, check how many duplications it has, and then we obtain one histogram item. Ultimately, we obtain the histogram for the residue, still denoted as $f(x)$ which will be used again.

2.12. At this point, we have repeated the above process until no further duplication can be found. In each loop we obtained a bag (or multiset) of duplications, and now we add them together to get the bag that contains all the duplications, which is exactly the multiset representation of one assembly pathway.

2.13. Lastly, based on the multiset representation, we can calculate the index of this pathway. 2.14. Run 2.1 - 2.13 for Nstep2 (a predefined parameter) number of times, to find the pathways 
that have the lowest pathway index.

\subsection{Executable program manual (AssemblyMC.exe)}

Download zip file AssemblyMC.zip. Unzip it and you will see six files:

1. AssemblyMC.exe, the executable file to calculate the shortest assembly pathways for a single molecule, which runs on Windows 10 .

2. libinchi.dll, a dynamic-link library file that is necessary for the exe file to run. It must be in the same folder as AssemblyMC.exe.

3. readme.pdf, the manual of the program.

4. Adenine.mol, the mol file (a standard file format to hold the information of a molecule) of the molecule adenine. It is the input of the exe file if you wish to calculate the shortest assembly pathways for adenine. Mol files of molecules can be freely downloaded from online databases such as ChEMBL, PubChem, etc.

5. example_Adenine_pathway.txt, one file of the results, which will be explained later.

6. example_Aenine_histogram.txt, one file of the results, which will be explained later.

Keep them in the same folder. Then:

1. Download the mol file of the molecule that you wish to calculate, and put it into the same folder of AssemblyMC.exe (here we will just take Adenine.mol as an example).

2. Open Windows command prompt (cmd.exe), and navigate to this folder.

3. Enter >AssemblyMC.exe Adenine.mol and it will then analyze the file Adenine.mol, namely, molecule adenine.

There are two optional parameters. The first parameter (Nstep2, referring to SI section 3.2) specifies how many possible assembly pathways to try in order to find the shortest one (1 is the default value, meaning there is no limit); while the second parameter (Nstep1, referring to SI section 3.2) means how many fragmenting schemes to try to obtain the 
fragments histogram ( -1 is the default value, meaning either $1 \%$ of all possible fragmenting schemes, or 100000, whichever is greater).

The example above uses no parameter. The following example uses both parameters

\section{$>$ AssemblyMC.exe Adenine.mol 50000100000}

which we will take as an example to show what to expect when it runs and after it finishes. It first calculates the fragments histogram, and shows the process as

Please wait...
.100000 steps to try in step $1 .$.
$>23132>45035>67144$

When the number (e.g., >67144) shown equal to the second parameter, this process to obtain the fragments distribution (Monte Carlo Step 1, referring to Fig. S2) finishes. And the message below will be displayed in the prompt:

$>23132>45035>67144>90217$
$=============================$
$======$ Step 1 is Done. $======$
$==========================$

After that, it will start Monte Carlo Step 2 (Fig. S2) immediately. It will keep displaying the symbol ">" until 10000 pathways have been tried. Then, in the same folder, a file Adenine_pathway.txt will be created that is the ultimate result and contains all the information we need, which we will explain how to interpret soon (it will also display some texts in the prompt, which basically repeats what is written in the file Adenine pathway.txt). Note that the program will continue to run until it has tried Nstep2 (the first parameter you input) number of possible assembly pathways; but each time it has tried 10000 pathways, it will update and overwrite the file Adenine_pathway.txt. If the first parameter you input is the default value -1, you may close the program manually when you think it has tried enough possible pathways. 
Now we will explain how to interpret the results contained in Adenine_pathway.txt. We will take the file example_Adenine_pathway.txt as an example to explain due to the fact that this method is Monte Carlo (thus contains randomness) so there might be slight differences at each time it runs. Indeed, example_Adenine_pathway.txt is the file Adenine_pathway.txt generated for a particular run, and we just renamed it as example_Adenine_pathway.txt.

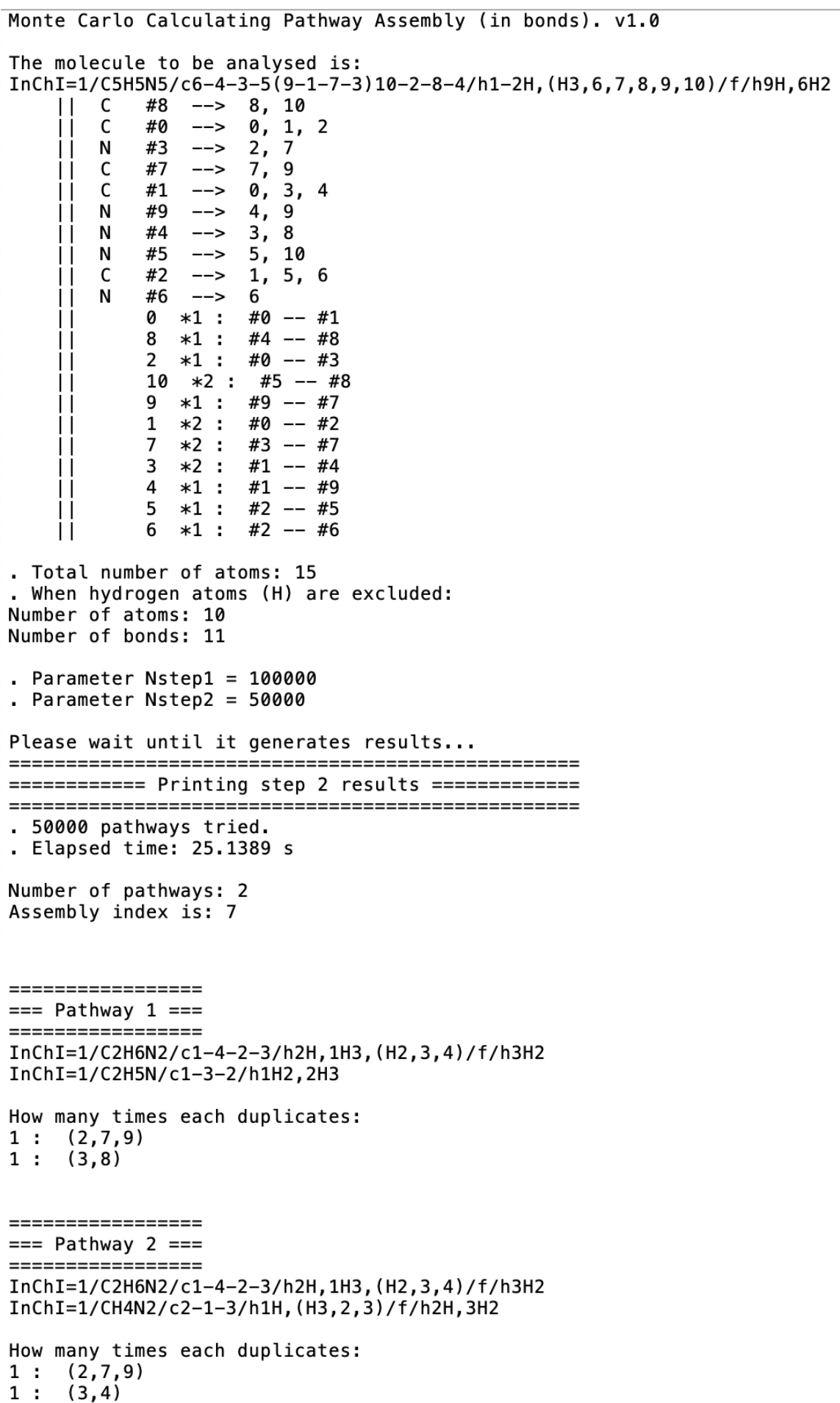

- Total number of atoms: 15

- When hydrogen atoms (H) are excluded:

Number of atoms: 10

Number of bonds: 11

- Parameter Nstep1 $=100000$

- Parameter Nstep2 $=50000$

Please wait until it generates results...

$===$

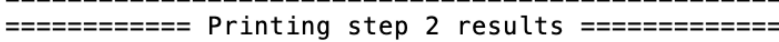

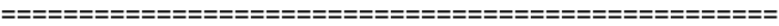

- 50000 pathways tried.

- Elapsed time: 25.1389 s

Number of pathways: 2

Assembly index is: 7

$==============$

=== Pathway 1 ===

InChI=1/C2H6N2/c1-4-2-3/h2H, $1 \mathrm{H} 3,(\mathrm{H} 2,3,4) / \mathrm{f} / \mathrm{h} 3 \mathrm{H} 2$

InChI $=1 / \mathrm{C} 2 \mathrm{H} 5 \mathrm{~N} / \mathrm{c} 1-3-2 / \mathrm{h} 1 \mathrm{H} 2,2 \mathrm{H}$

How many times each duplicates:

$1:(2,7,9)$

$1:(3,8)$

$===$ Pathway 2 ==

$====$

InChI $=1 / \mathrm{C} 2 \mathrm{H} 6 \mathrm{~N} 2 / \mathrm{c} 1-4-2-3 / \mathrm{h} 2 \mathrm{H}, 1 \mathrm{H} 3,(\mathrm{H} 2,3,4) / \mathrm{f} / \mathrm{h} 3 \mathrm{H} 2$

InChI $=1 / \mathrm{CH} 4 \mathrm{~N} 2 / \mathrm{c} 2-1-3 / \mathrm{h} 1 \mathrm{H},(\mathrm{H} 3,2,3) / \mathrm{f} / \mathrm{h} 2 \mathrm{H}, 3 \mathrm{H} 2$

How many times each duplicates:

$1:(2,7,9)$

$1:(3,4)$ 
The block started with "I " records how the molecule is represented in the program. The lines starting with capital letters represent atoms. For example, the first line " $\mathrm{C} \# 8$--> 8, 10" means the carbon (C) atom's ID is 8 (denoted by symbol \#), and it is attached by bond 8 and 10; the seventh line "N \#4 --> 3, 8" means the nitrogen (N) atom's ID is 4, and it is attached by bond 3 and 8 . The lines starting with integers represent bonds. For example, the first line " $0 * 1: \# 0$ -- \#1" means this bond's ID is 0 , which is a single bond (denoted by symbol *), and it connects atom 0 and atom 1 ; the fourth line "10 $* 2: \# 5$-- \#8" means this bond's ID is 10 , which is a double bond, and it connects atom 5 and atom 8 . All other information is self-evident.

Now let's look at the lines "Number of pathways: 2" and "Assembly index is: 7". It tells you that the assembly number (MA) of this molecule adenine is 7 (namely, the assembly index of the shortest assembly pathways of adenine), and there are two assembly pathways with this MA, each of which is displayed in the following lines, starting with "=== Pathway $i==="$. Take the first pathway as an example, each line started with "InChI=" is an InChI (International Chemical Identifier) of a chemical structure. The first line after "How many times each duplicates:", i.e., "1 : $(2,7,9)$ " means that in the multiset representation of this pathway (referring to Fig. $2 d$ in the main text) the multiplicity is 1 for the first structure listed above "InChI=1/C2H6N2/c1-4-2-3/h2H,1H3,(H2,3,4)/f/h3H2" which is made of bond 2, 7 and 9. The second line " $1:(3,8)$ " means that in the multiset representation the multiplicity is 1 for the second structure "InChI $=1 / \mathrm{C} 2 \mathrm{H} 5 \mathrm{~N} / \mathrm{c} 1-3-2 / \mathrm{h} 1 \mathrm{H} 2,2 \mathrm{H} 3$ " which is made of bond 3 and 8 . Therefore, the multiset representation of assembly pathway 1 is $\{\mathrm{InChI}=1 / \mathrm{C} 2 \mathrm{H} 6 \mathrm{~N} 2 / \mathrm{c} 1-4-2-$ 3/h2H,1H3,(H2,3,4)/f/h3H2, InChI=1/C2H5N/c1-3-2/h1H2,2H3 \}. InChI can be easily transformed to any other format by standard software (e.g., OpenBabel). Here we use OpenBabel to transform the two InChI's into the graph representation of molecules, so this pathway can also be denoted as: 
$\left\{\mathrm{H}_{\mathrm{N}} \rightarrow \mathrm{H}^{\mathrm{NH}_{2} \mathrm{C}}-\mathrm{N} \gtrless_{\mathrm{CH}_{2}}\right\}$

Note that we should ignore all of the hydrogens as we have ignored them from the beginning (these hydrogens appear in the graphs because we have to use them as some "placeholders" to generate proper InChI's).

Likewise, the multiset representation of assembly pathway 2 is $\{\mathrm{InChI}=1 / \mathrm{C} 2 \mathrm{H} 6 \mathrm{~N} 2 / \mathrm{c} 1-4-2-$ 3/h2H,1H3,(H2,3,4)/f/h3H2, InChI=1/CH4N2/c2-1-3/h1H,(H3,2,3)/f/h2H,3H2 \}, or denoted as:<smiles>CN=CN</smiles>

Lastly, there is another temporary file Adenine_histogram.txt also generated, that is automatically used by the program in Monte Carlo Step 2, so do not delete it. It displays the fragments histogram obtained in Monte Carlo Step 1. It might be useful to the reader, so we will also explain it (we will take example_Adenine_histogram.txt as an example). The first part of this file is the same as example_Adenine_pathway.txt, while the second half is written as follows. It displays fragments in groups (every five fragments constitute a group) which are sorted by the number of occurrences (namely, y-axis value of the fragments distribution, referring to SI section 3.1 to learn more about this distribution). So, here we see that structure InChI $=1 / \mathrm{C} 2 \mathrm{H} 5 \mathrm{~N} / \mathrm{c} 1-2-3 / \mathrm{h} 2 \mathrm{H}, 1,3 \mathrm{H} 2 \quad$ appears three times, $\mathrm{InChI}=1 / \mathrm{CH} 4 \mathrm{~N} 2 / \mathrm{c} 2-1-$ 3/h1H,(H3,2,3)/f/h2H,3H2 appears three times, and so on; structure $\mathrm{InChI}=1 / \mathrm{C} 3 \mathrm{H} 5 \mathrm{~N} / \mathrm{c} 1-3-4-$ 2/h3H,1-2H2 appears twice, InChI=1/C2H6N2/c1-4-2-3/h2H,1H3,(H2,3,4)/f/h3-4H appears twice, and so on. 


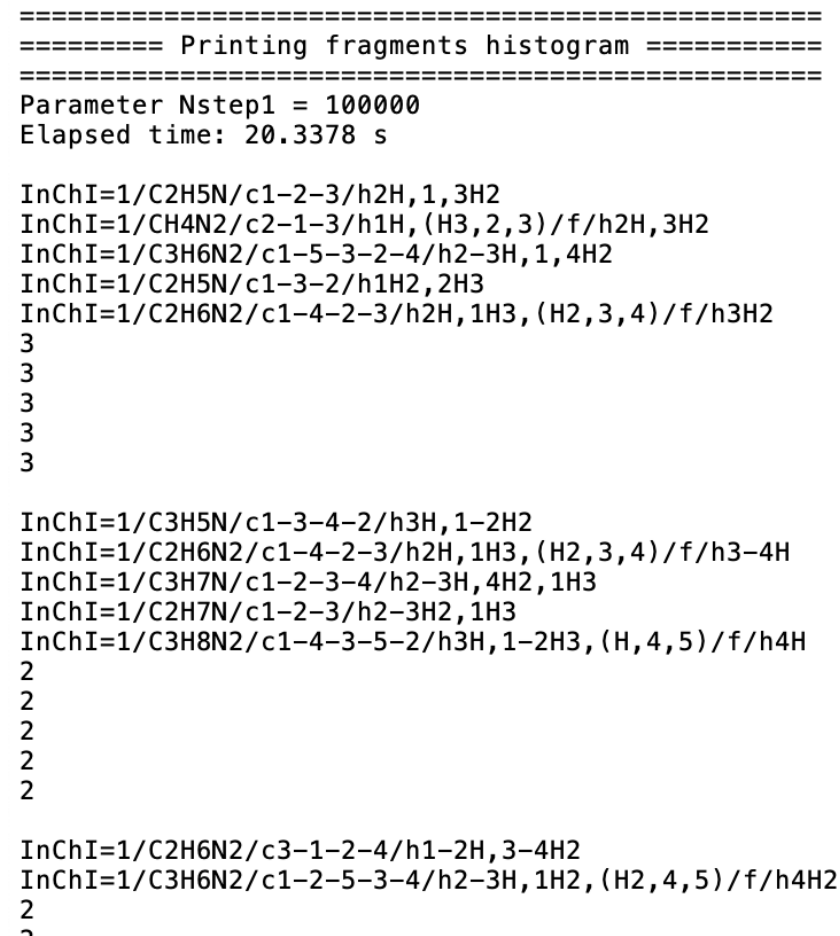

\subsection{Examples: several molecules' shortest assembly pathways calculated}

Here we apply AssemblyMC.exe to five molecules as examples. We always set the parameters Nstep $1=100000$ and Nstep $2=10000$. The first example is aspirin. The command we run is

\section{>AssemblyMC.exe Aspirin.mol 10000100000}

From the output of this program (see SI section 3.3 for details), we know that aspirin's MA is 8 and one of the shortest assembly pathways in multiset representation is as follows:<smiles>CC(=O)Oc1ccccc1C(=O)O</smiles><smiles>C=CC</smiles>

Note that we should ignore all of the hydrogens as we have ignored them from the beginning (these hydrogens appear in the graphs because we have to use them as some "placeholders" to generate proper InChI's). 
Likewise, the 2nd example is hexachlorobenzene. Its MA is 5, and one of its shortest assembly pathways is:

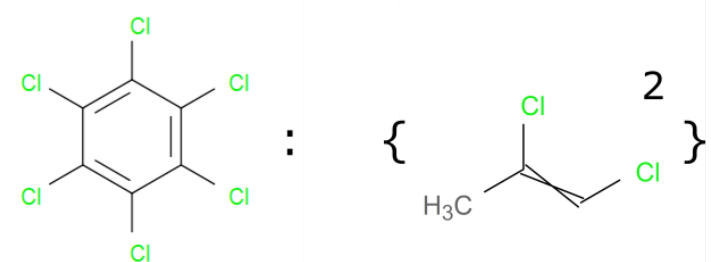

The 3rd example is tryptophan. Its MA is 11 , and one of its shortest assembly pathways is:

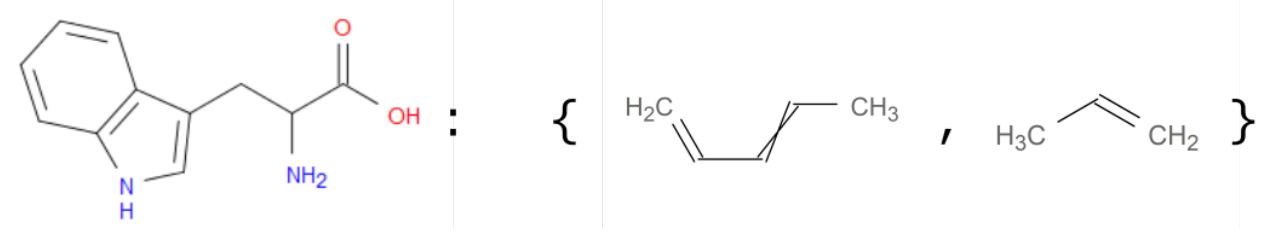

The 4th example is 5-[(1-Carboxyvinyl)oxy]-4-hydroxy-3-(phosphonooxy)-1-cyclohexene-1carboxylic acid. Its MA is 14 , and one of its shortest assembly pathways is:

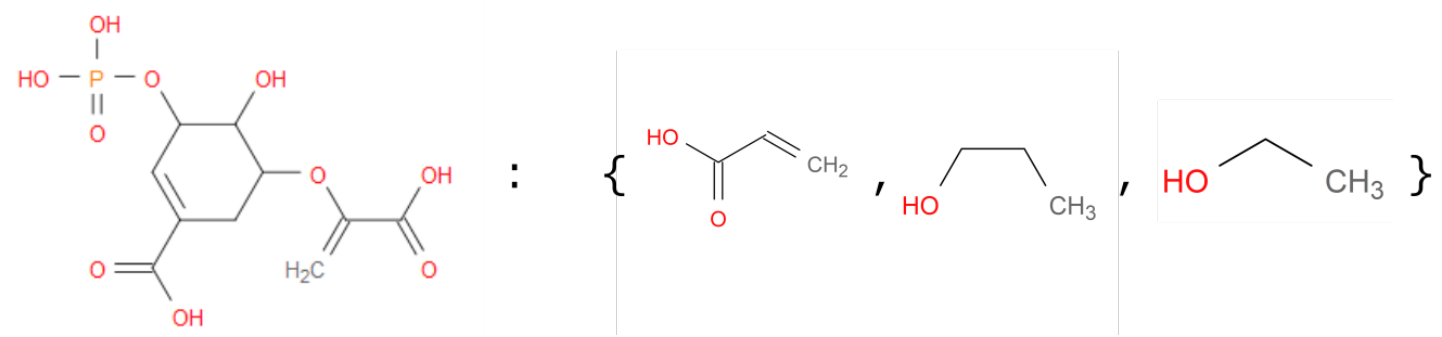

The 5th example is sildenafil, or commonly known as the brand name Viagra. Its MA is 25, and one of its shortest assembly pathways is:

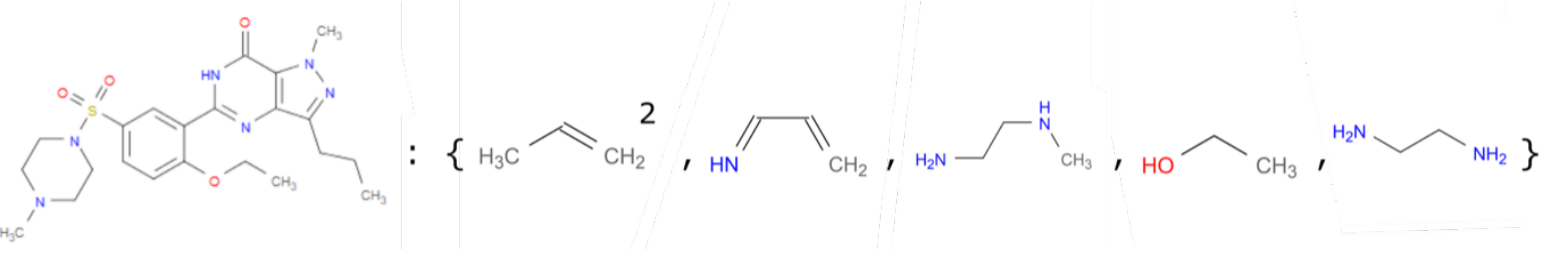




\section{Monte Carlo algorithm: for molecular assembly tree}

\subsection{Extension to a group of molecules}

The main text and SI section 3 explained how AssemblyMC.exe computes the shortest assembly pathways of a single molecule. As mentioned in the main text, to compute the molecular assembly tree of a group of molecules (equivalently, the shortest assembly pathways to construct all of these molecules simultaneously), we only need to consider this group of molecules as a single "big molecule" with disconnected parts, which are the individual molecules. If in the beginning of the program AssemblyMC.exe we create an object that stores the complete information of this big molecule with the same format as the object that is used to store the information of a single molecule, this program can then compute the shortest pathways automatically and nothing needs to be changed after this first step.

More specifically, in AssemblyMC.exe, it first reads the mol file of the single molecule in question, and construct a "molecule" object ( $M O L_{-}$BOND class), which stores all of the atoms and bonds, also giving each atom an index and each bond (connecting two atoms) an index (see SI section 3.2). For a group of molecules, after creating the $M O L_{-} B O N D$ object that stores the first molecule's information, it should immediately read the second molecule's mol file, and then add atoms and bonds of the second molecule to the previous $M O L_{-} B O N D$ object. Note that the atom (or bond) index should start from the total number of atoms (or bonds) $M O L_{-} B O N D$ already has, instead of starting from 0 . It should continue to read mol files and add atoms and bonds, until all mol files have been read. Finally, the $M O L_{-} B O N D$ object that stores the information of this big molecule can be obtained. What follows is the same process as AssemblyMC.exe. For convenience, we made a standalone executable file (TreeMC.exe, see the manual in the next subsection) to compute the assembly tree of a group of molecules. 
Although the input and output have different formats to AssemblyMC.exe, the basic logic of the two programs is the same.

\subsection{Executable program manual (TreeMC.exe)}

Download zip file TreeMC.zip. Unzip it and you will the following files:

1. TreeMC.exe (runs on Windows), the executable file to calculate the molecular assembly tree for a group of molecules.

2. libinchi.dll, a dynamic-link library file that is necessary for the exe file to run. It must be in the same folder as TreeMC.exe.

3. readme.pdf, the manual of the program.

4. Mol files: Adenine.mol, Guanine.mol, Thymine.mol, Cytosine.mol and Uracil.mol, the mol files (a standard file format to hold the information of a molecule) of nucleobases which will be used as the example. They are the required files if you wish to compute the molecular assembly tree of these five nucleobase types. Mol files of molecules can be freely downloaded from online databases such as ChEMBL, PubChem, etc.

5. ToDo.txt, the required user-defined file to feed the exe file, which will be explained in details later.

6. Generated temporary files: example_Adenine_histWhole.txt, example_Adenine _histWhole.txt, example_Adenine_histWhole.txt, example_Adenine_histWhole.txt and example_Adenine_histWhole.txt, the temporary files generated by the program when it is running, which will be explained later.

7. Generated result files: example_Tree_AllPaths.txt, example_Tree_bySize.txt and example_Tree_byRepeats.tx, the files generated at the end that contains all the information we need, which will be explained later. 
Now we will show how to run the program, in order to calculate the molecular assembly tree of the five nucleobase types: adenine, guanine, thymine, cytosine and uracil. As described in the main text, it is equivalent to calculate the shortest assembly pathways for these molecules.

1. Put the mol files of these five nucleobases in the folder as TreeMC.exe. In general, you could download these mol files from online databases, but here we have already downloaded them for you.

2. Create a plain-text file named ToDo.txt, based on the following format strictly (here we have created it for you):

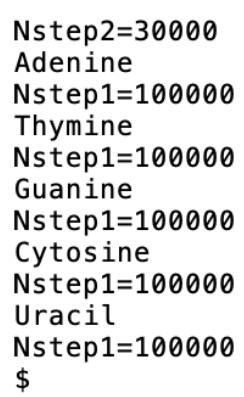

The first line is "Nstep2=XXX" where $\mathrm{XXX}$ is the parameter Nstep2 for the program, meaning how many possible assembly pathways to try in order to find the shortest one (referring to SI section 3.2). The larger Nstep2 is, the more accurate the final result will be (but it takes longer time to run). The second line is the name of one mol file: Here it is "Adenine" because the mol file is Adenine.mol (note that if the mol file is abc.mol, the second line should be "abc"). The third line is "Nstep1=XXX" where XXX is the parameter Nstep1 for this molecule, meaning how many fragmenting schemes to try to obtain the fragments histogram for this molecule (referring to SI section 3.2). The larger Nstepl is, the more accurate the result will be (but it takes longer time to run). But in any case, we recommend that Nstepl should be at least 100000. The 4th and 5th lines are for another molecule (here it is for thymine); the 6th and 7th lines are for another (here it is guanine); and so on. Note that the order of the molecules does not matter, and Nstepl can be different for different molecules. Finally, the last line is the symbol "\$". 
Note that any Nstep1 could be a special value "0", for example,

Adenine

Nstep $1=0$

which means that the program will use the already-existing histogram file Adenine_histWhole.txt instead of calculating it from scratch. If Nstepl is not 0 , then Adenine_histWhole.txt is a temporary file (describing the fragments distribution, see details later) that will be generated when the program is running, and will be used again automatically until the program finishes. If Nstepl is set to 0 , then the program will not generate / overwrite the file Adenine_histWhole.txt, but directly use Adenine_histWhole.txt to calculate the tree, which may save a lot of time (in this case, if there is no such file named Adenine_histWhole.txt in this folder, the program will report an error and terminate).

3. Simply double click TreeMC.exe to run, and wait it to finish, which may take a while.

As the program runs, progress is displayed on screen as follows for Monte Carlo Step 1 (referring to Fig. S2):

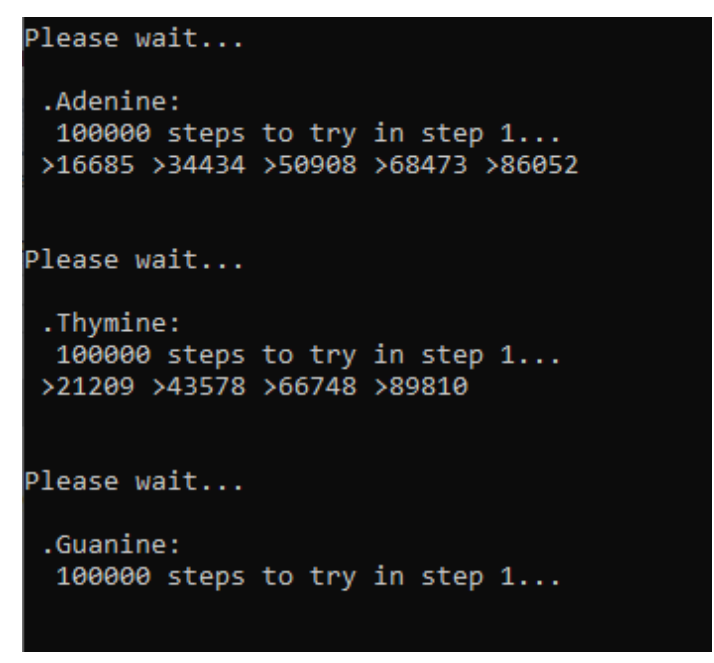

Every time when it obtains the fragments distribution for a molecule, a file named $X X X \_$histWhole.txt will be generated or overwritten if already existed, where $X X X$ is the name of the corresponding mol file. Just leave them there and they will be automatically reused in 
Monte Carlo Step 2. When each molecule's fragments distribution have been obtained, it will display a message, and Monte Carlo Step 2 will start immediately:

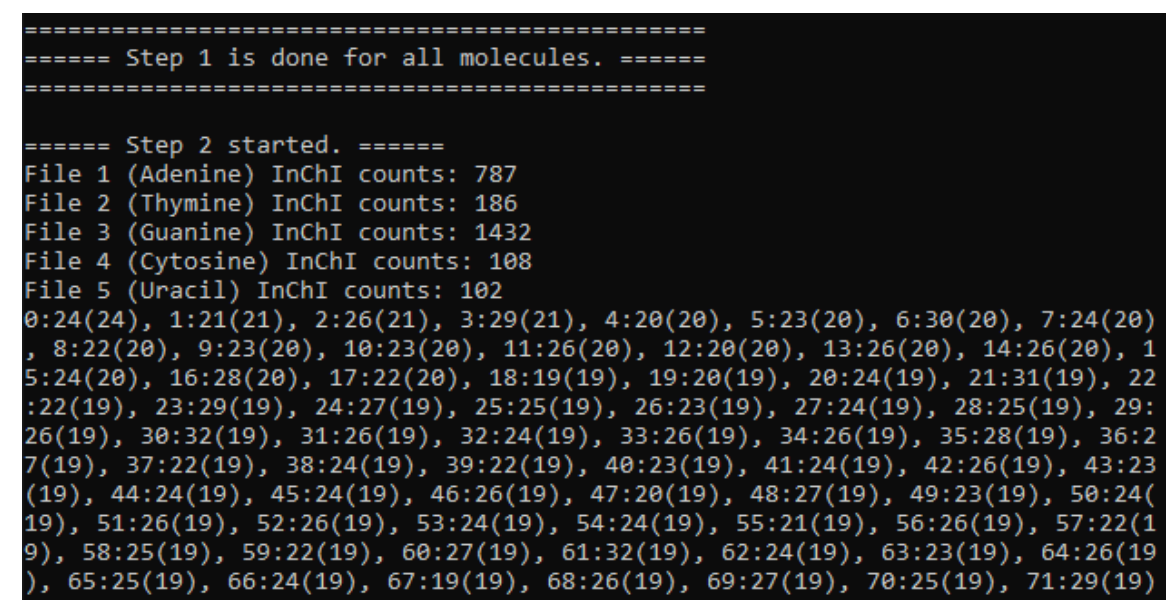

The message " $71: 29$ (19)", for example, means that 71 possible pathways have been tried, the assembly index of the previous pathway is 29 , and the minimum index till now is 19 . This will continue until it checks Nstep2 number of possible pathways. After that, a message will be displayed (as follows, which means that there are 5 pathways having index 16; 52 pathways having index 17; and so on) and the program finishes (result files will be generated).

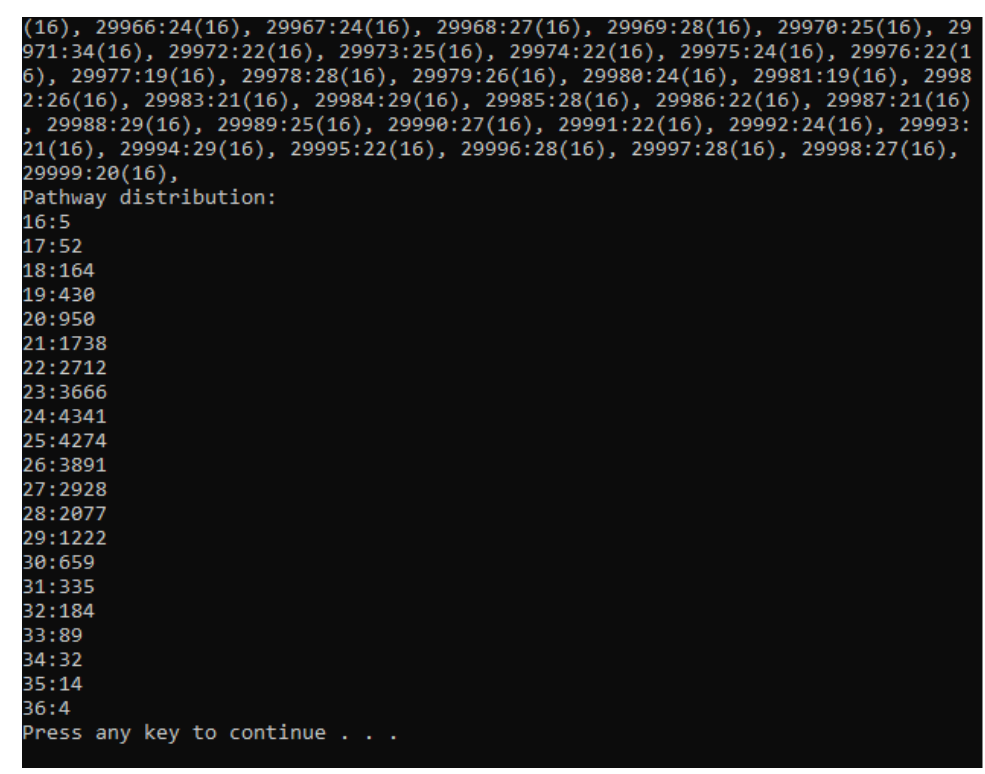

Now we will explain how to interpret the result files. The file Tree_AllPaths.txt is one of the three files generated at the end, which contains all the information we need. Here we will take 
example_Tree_AllPaths.txt as an example to explain due to the fact that this method is Monte Carlo (thus contains randomness) so there might be slight differences at each time it runs. Indeed, example_Tree_AllPaths.txt is the file Tree_AllPaths.txt generated for a particular run, and we just renamed it as example_Tree_AllPaths.txt. This is how the file starts with:

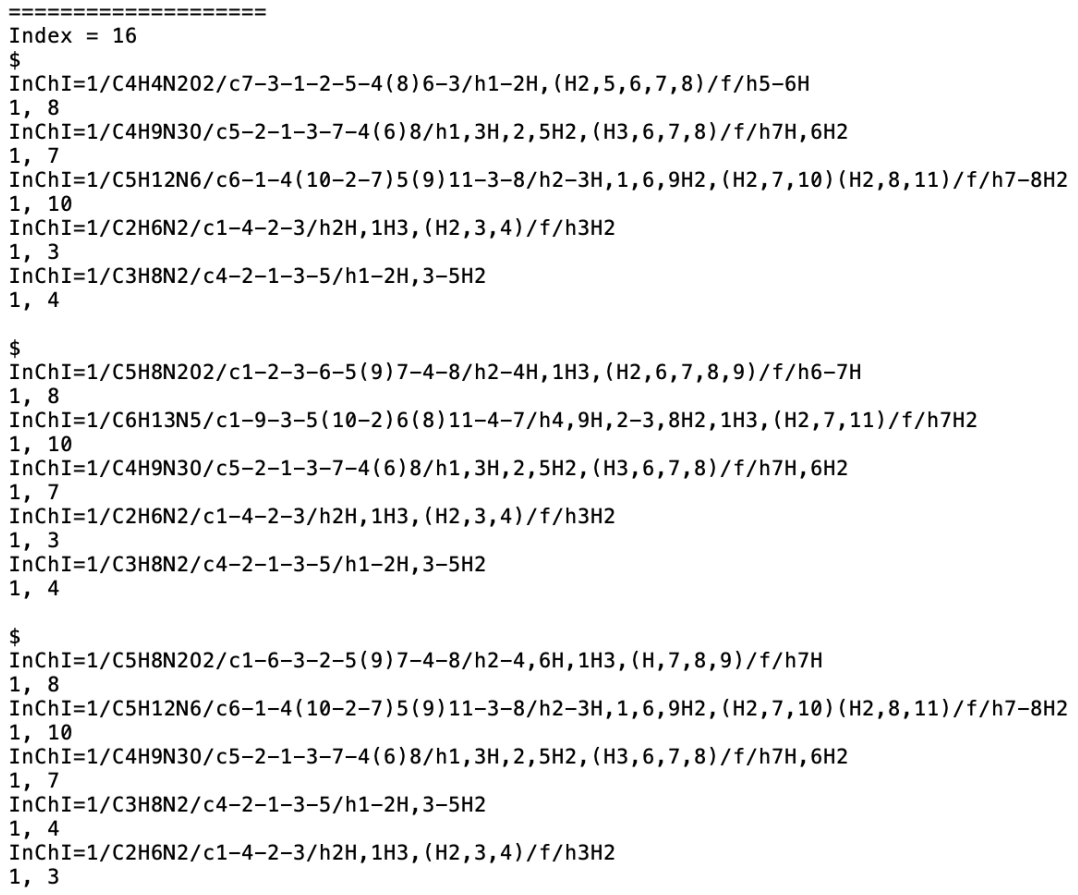

The assembly pathways having assembly index 16 are the shortest, so they are shown first. There are 5 pathways having index 16, so they are shown one by one here (it is a long text file, and it shows only 3 pathways here. After index 16, it shows all pathways having index 17, and then 18 , and so on). Each pathway stars with the symbol "\$". Each pathway is made of chemical structures, represented by these InChI (International Chemical Identifier) strings. The two numbers below each InChI means the counts and the number of bonds of this structure respectively. We see that the first pathway shown above has five InChI's, corresponding to the following five chemical structures respectively (we used one of the standard software OpenBabel to transform the InChI's into graph representations of molecules): 
<smiles>NCC/C=C\NC(N)=O</smiles><smiles>NCN=C(CN)C(N)NCN</smiles><smiles>CN=CN</smiles>

We can also confirm that the first structure has 8 bonds, the second has 7 bonds, and so on. Note that we should ignore all of the hydrogens as we have ignored them from the beginning (these hydrogens appear in the graphs because we have to use them as some "placeholders" to generate proper InChI's). Therefore, the first pathway can be written as the multiset represented:

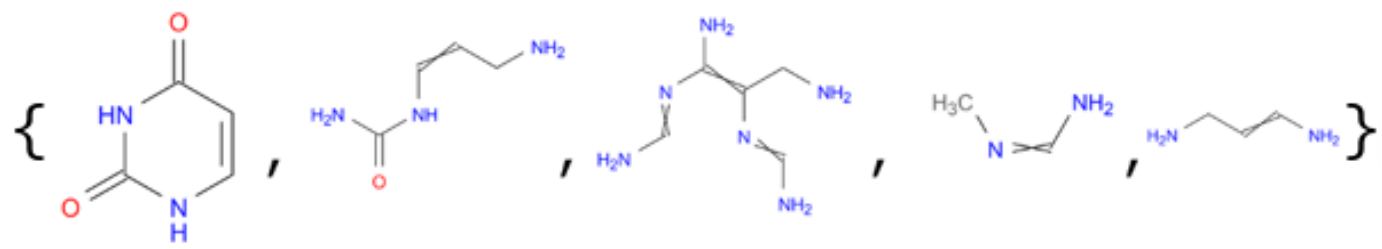

Equivalently, it is the molecular assembly tree of adenine, guanine, thymine, cytosine and uracil (written in multiset representation). We can then manually draw the molecular assembly tree of them (see details in SI section 4.3):<smiles>Nc1ncnc2[nH]cnc12</smiles><smiles></smiles><smiles>Cc1c[nH]c(=O)[nH]c1=O</smiles><smiles>CC=N/C(CN)=C(N=CN=CN)/N=C/N</smiles><smiles>Nn1c(=O)cc[nH]c1=O</smiles><smiles>Nc1cc[nH]c(=O)n1</smiles> 
Another file generated at the end is Tree_bySize.txt (here we take example_Tree_bySize.txt as the example). The information is written as InChI, two numbers, InChI, two numbers, InChI, two numbers, ... The first number below an InChI means how many assembly pathways in the file Tree_AllPaths.txt contain this chemical structure; and the second number is the number of bonds of this structure. The structures are sorted by the number of bonds (namely the second number). The last file generated at the end is Tree_byRepeats.txt (here we take example_Tree_byRepeats.txt as the example). It is almost the same as the file Tree_bySize.txt, but the structures are sorted by the first number. Some readers may be interested in these information, that's why we generated them.

Finally, we will explain the temporary files (namely, the histogram files, e.g., Adenine_histWhole.txt) generated when the program is running. We will take example_Adenine_histWhole.txt as an example. The block started with "I I" records how the molecule is represented in the program. The lines started with capital letters represent atoms. For example, the first line " $\mathrm{C} \# 8$--> 8, 10" means the carbon (C) atom's ID is 8 (denoted by symbol \#), and it is attached by bond 8 and 10; the seventh line " $\mathrm{N} \# 4-->3,8$ " means the nitrogen (N) atom's ID is 4, and it is attached by bond 3 and 8 . The lines started with integers represent bonds. For example, the first line “ $0 * 1: \# 0$-- \#1" means this bond's ID is 0 , which is a single bond (denoted by symbol *), and it connects atom 0 and atom 1 ; the fourth line " 10 *2 : \#5 -- \#8" means this bond's ID is 10, which is a double bond, and it connects atom 5 and atom 8 . The information below the symbol " $\$$ " is displayed as: 


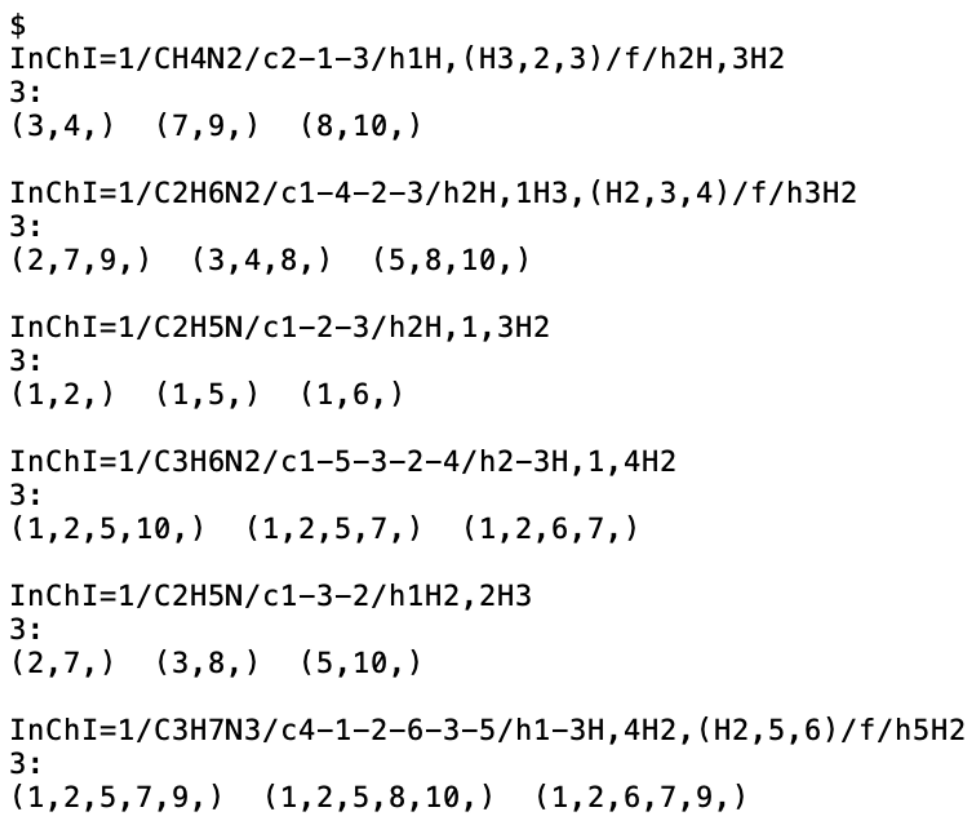

Each InChI is a fragment of this molecule, namely, a chemical structure. The number below means how many of this identical structure are contained in this molecule. For example, adenine contains 3 identical structures "InChI=1/CH4N2/c2-1-3/h1H,(H3,2,3)/f/h2H,3H2". Each of the three brackets below shows which bonds constitute this structure. These temporary files are generated because we may only need to calculate the fragments distribution once. For example, if next time we want to calculate the molecular assembly tree of adenine and other molecules, we can put this already-calculated histogram file there, and set Nstepl for adenine to 0 , the program will then directly take this file rather than calculate it from scratch.

\subsection{Visualize molecular assembly tree (based on multiset representation)}

Here we take the assembly tree of A, G, T, U and C as an example (Fig. $4 \mathrm{~b}$ in the main text). First of all, we run TreeMC.exe on the five molecules and ultimately obtain the multiset representation of one of the shortest assembly pathways, as shown below (see SI section 4.2 for the manual). 


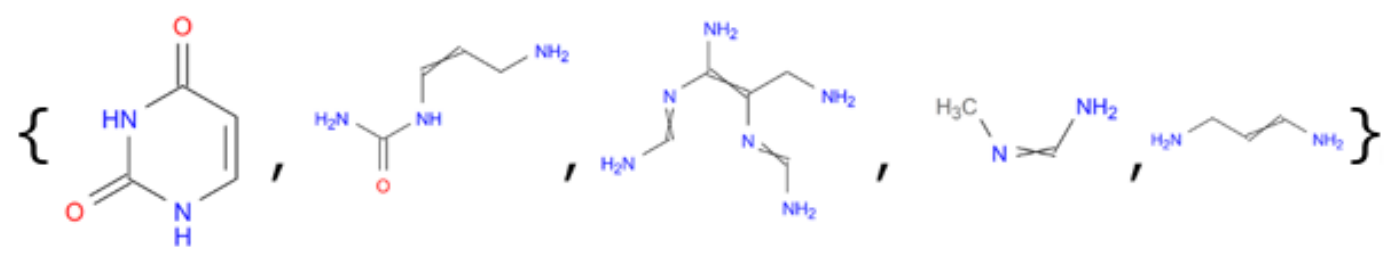

This multiset will then be used to draw the assembly trees of AGTUC. For convenience, we denote the five structures in turn as [13] (which is also U), [12], [11], [2] and [10] (to be consistent with Fig. $4 \mathrm{~b}$ in the main text). It's more convenient to start with larger structures. We first see that $\mathrm{T}$ is made of [13] and a C-C bond, so we link [13] to T (referring to Fig. 4b). In principle, we should also link $\mathrm{C}-\mathrm{C}$ to $\mathrm{T}$, but for a better visualization, we will always omit the links from the basic building blocks, i.e., individual bonds. Second, we see that [13] is made of [12] and a $\mathrm{C}=\mathrm{O}$ bond and that cytosine is made of [12] and a $\mathrm{C}=\mathrm{N}$ bond, so we link [12] to both [13] and cytosine (hence a fork starting from [12]). Third, we see that A is made of [11] and a $\mathrm{C}=\mathrm{N}$ bond and that $\mathrm{G}$ is made of [11], a $\mathrm{C}-\mathrm{N}$ bond and a $\mathrm{C}=\mathrm{O}$ bond, so we link [11] to both A and G. Fourth, we see that [12] is made of [10] and three individual bonds, so we link [10] to [12]. Fifth, we see that [11] is made of [10] and two of [2], so we link [10] to [11], and also link [2] to [11] (in Fig. 4b we drew two arrows from [2] to [11] to represent that [11] contains two of [2], but in general, we may just draw one, especially when the tree is big). Then we can see that [2] and [10] are just made of individual bonds (since there is no key assembly building blocks left in the multiset that have not been used), so the linking process is finished.

In fact, till now, the complete information of the tree is there, i.e., the mathematical graph consisting of nodes (structures / molecules) and edges (links). Next we employ an extra principle to arrange all the nodes: we put all nodes at different levels, and a node is always placed one level above its constituted nodes (if a node is made of several nodes, it is placed one level above its constituted node with the highest level). Finally, we obtained Fig. 4b. 
There is one more remark. Although the Monte Carlo algorithm generates good-enough result, it is not guaranteed to find the shortest assembly pathway. The more Monte Carlo steps the algorithm tries, the shorter pathways it finds. But by using the suggested parameters in this paper, the results are good enough. For example, the ten opiates in Fig. 8 in the main text, which in total have 268 bonds, are certainly a large group of complex molecules; and the final output from one run of the Monte Carlo algorithm shows that the smallest MA is 105, which is already much smaller than the number of bonds (a good-enough result, see below):

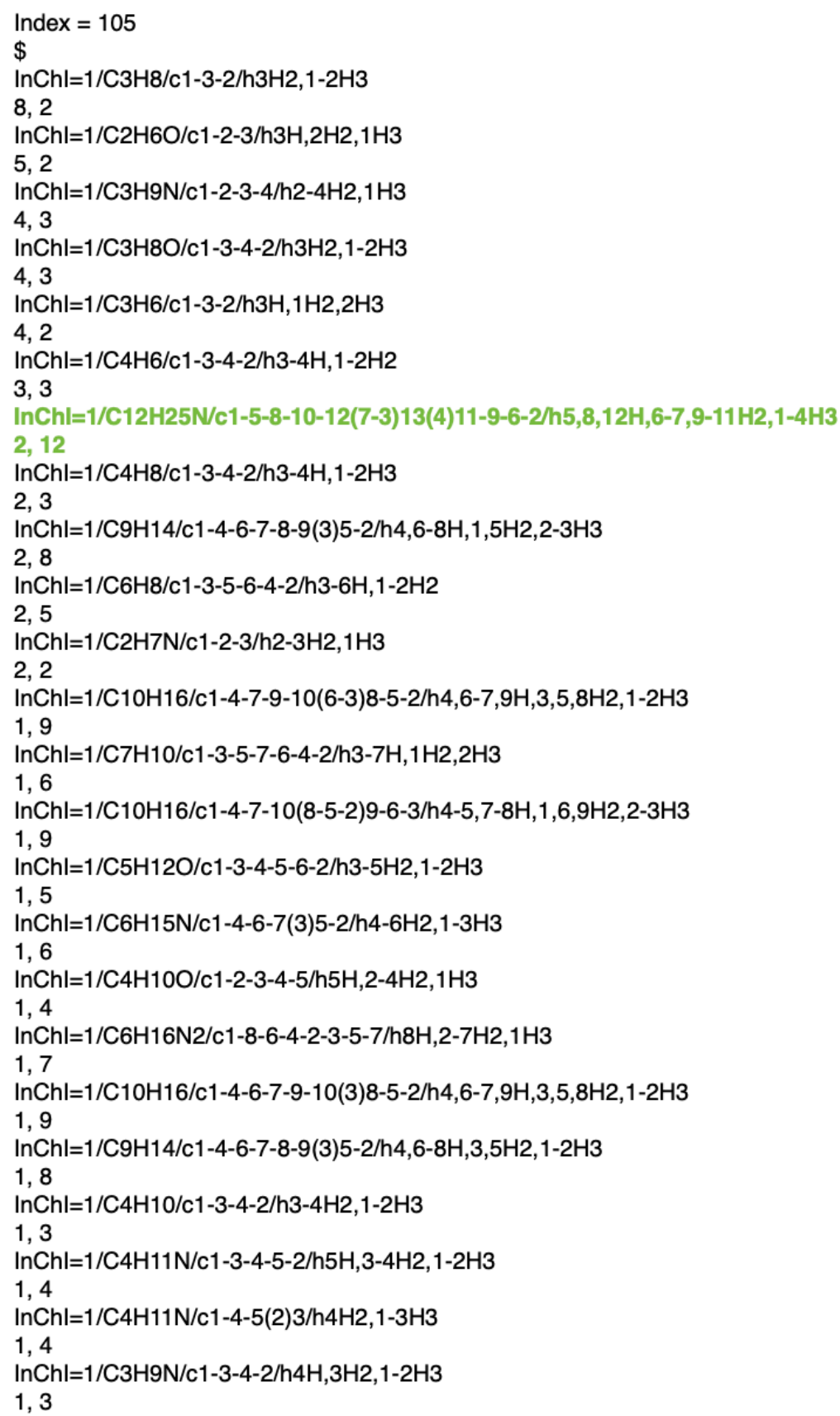


Nevertheless, for a large group of complex molecules, we may use human intuitions to improve the final results. We see from the InChI's above that structure [14] (figure below, which is the highlighted InChI above) is included in this assembly pathway and is shared by two molecules, morphine and thebaine. But evidently, there is a larger shared structure [15] (figure below) that completely contains [14] but the algorithm failed to find (might be able to find it in other runs). So, if we replace [14] with [15] in the multiset, we can certainly have a shorter pathway. You may find more such larger shared structures that lead to shorter pathways. Indeed, we have used this type of intuitions to draw Fig. 8. The bottom line is that, although the Monte Carlo algorithm may not be able to find the "ultimate" shortest pathways (since the problem itself is extremely hard when the size of the molecules is large, at least as hard as a NP-complete problem, see SI section 2), it is able to find short enough pathways, which might be further used as the bases to work out even shorter pathways.

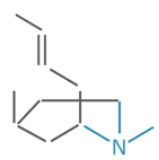

[14]

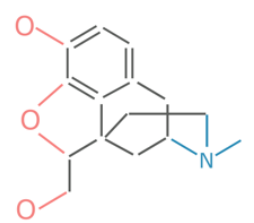

[15]

\section{Generate alternated molecules using MOLGEN}

To estimate the size of a constrained subset of theoretical chemical space, we used the commercial software MOLGEN 5.0, which enumerates all structures for a given molecular formula, or formula range. MOLGEN commands we used to enumerate molecules took the form:

$$
>\text { mgen } \mathrm{C} 6 \mathrm{H6}-\mathrm{v}
$$

which will enumerate all structural isomers with molecular formula $\mathrm{C} 6 \mathrm{H} 6$, or

$$
>\text { mgen } \mathrm{C} 0-10 \mathrm{~S} 0-10 \mathrm{~N} 0-1000-10 \mathrm{H} 0-100-\operatorname{sum} \mathrm{C}+\mathrm{N}+\mathrm{O}+\mathrm{S}=10
$$


which will enumerate all structural isomers with up to 10 atoms of each of $\mathrm{C}, \mathrm{N}, \mathrm{O}$, and $\mathrm{S}$, and with a total of 10 atoms of $\mathrm{C}, \mathrm{N}, \mathrm{O}$, and $\mathrm{S}$, and up to $100 \mathrm{H}$ atoms (an arbitrary high figure chosen to represent any number of $\mathrm{H}$ atoms).

\section{Size of assembly space of the exemplified opiates}

There are two parameters for the Monte Carlo steps: Nstep1, meaning how many fragmenting schemes to try to obtain the fragments distribution, and Nstep2, meaning how many possible assembly pathways to try in order to find the shortest one (more details in SI section 3.2). Now we'll show how the size of the assembly space---the total number of unique duplicated fragments included in all assembly pathways calculated---of the exemplified opiates (section 5.3 in the main text) changes with Nstep1 and Nstep2, as shown in the figure below.

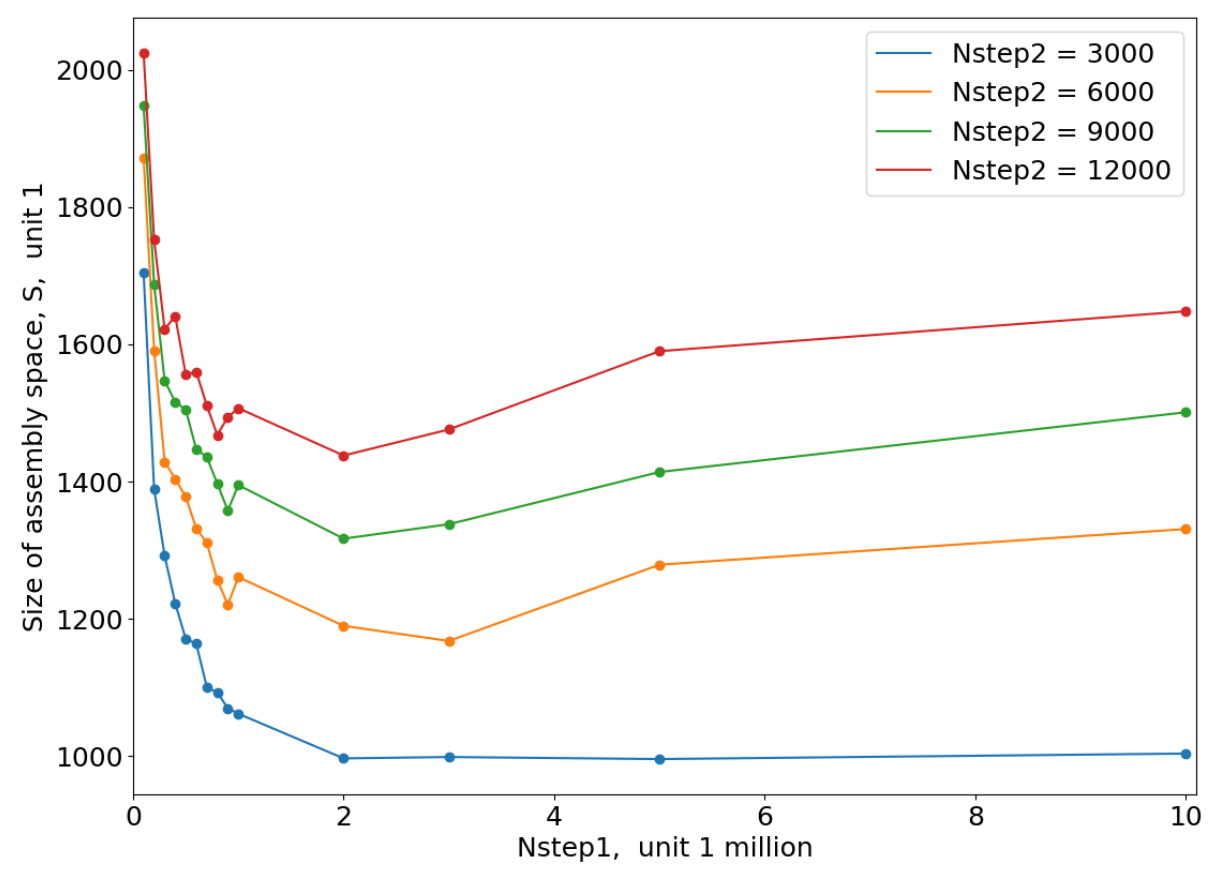

There is a trend that as Nstepl increases, the size of the assembly space $(S)$ first decreases sharply and then increase slowly. When Nstep 1 is small (for example, 0.5 million in this case), the fragment distribution would not be able to reflect the "real" distribution (as we use Monte Carlo approach to sample). The distribution is more spread out than it should be, i.e., the probability of drawing a fragment duplicated for a small number of times would be higher than 
it should be, while the probability of drawing a fragment duplicated for a large number of times would be lower than it should be. Therefore, in this case, the number of unique fragments that are drawn in all these processes would be larger than that in the case where the fragment distribution can reflect the "real" distribution. In this case, the threshold is around 2 million (as see from the figure above), after which the distribution is almost "real". Now, $S$ starts to increase, because the fragments that are duplicated for only very few times (twice, for example) have the chance to be be drawn, which is very unlikely before this threshold. The increase of $S$ is slow, because as long as this threshold is passed, the calculated fragment distribution is already quite close to the real one.

We also observed that the larger Nstep 2 is, the larger $S$ is. This is straightforward, because the more steps we try, the more unique fragments would be drawn. If Nstep2 goes to infinite, all unique fragments that are duplicated at least once would be drawn, i.e., the size of the assembly space then would be the number of all these unique fragments. But in this case, even when Nstep $2=3000$, the calculated assembly tree's MA (which is 105 ) is already very small, comparing to the number of bonds (which is 268). Therefore, all the unique fragments that have been drawn (namely, the assembly space obtained) already reflect the contingent information of these compounds in question, which is what we care about.

\section{SI References}

(1) D. E. Knuth, The Art of Computer Programming, Vol 2, "Seminumerical Algorithms" (3" edition), section 4.6 .3 (1997).

(2) Stuart M. Marshall, Douglas Moore, Alastair R. G. Murray, Sara I. Walker, Leroy Cronin, Quantifying the pathways to life using assembly spaces, arXiv:1907.04649 (2019). 
(3) Peter Downey, Benton Leong, Ravi Sethi, Computing sequences with addition chains, SIAM Journal on Computing, 10 (3): 638-646 (1981).

(4) Stephen A. Cook, The complexity of theorem-proving procedures, Proc. 3rd ACM Symposium on Theory of Computing, pp. 151-158 (1971). 